\title{
Xe adsorption on metal surfaces: First-principles investigations
}

\author{
Juarez L. F. Da Silva, ${ }^{1, *}$ Catherine Stampfl, ${ }^{1,2}$ and Matthias Scheffler ${ }^{1}$ \\ ${ }^{1}$ Fritz-Haber-Institut der Max-Planck-Gesellschaft, Faradayweg 4-6, D-14195 Berlin-Dahlem, Germany \\ ${ }^{2}$ The School of Physics, The University of Sydney, Sydney NSW 2006, Australia \\ (Received 15 March 2005; revised manuscript received 23 May 2005; published 16 August 2005)
}

\begin{abstract}
In the present paper we report in detail the results of our first-principles investigations of Xe/metal adsorption systems which were briefly presented in an earlier publication. We investigate Xe adsorption on the $\mathrm{Mg}(0001), \mathrm{Al}(111), \mathrm{Ti}(0001), \mathrm{Cu}(111), \mathrm{Pd}(111)$, and $\mathrm{Pt}(111)$ surfaces in the $(\sqrt{3} \times \sqrt{3}) R 30^{\circ}$ structure. Our calculations are based on density functional theory within the local-density approximation and generalized gradient approximation of Perdew, Burke, and Ernzerhof functions and employing the all-electron fullpotential linearized augmented plane wave method. From analysis of the lateral and perpendicular potentialenergy surfaces we find that Xe atoms adsorb in the on-top sites in all cases. We identify a noticeable site dependence of the Pauli repulsion, which largely actuates the adsorption site preference. We anticipate that the identified microscopic nature of the binding between Xe and metal atoms may also be valid for other rare-gas atom/metal systems.
\end{abstract}

DOI: 10.1103/PhysRevB.72.075424

PACS number(s): 68.43.Fg, 71.15.Mb, 71.15.Ap, 68.47.De

\section{INTRODUCTION}

Rare-gas atom adsorption on metal surfaces may be regarded as the paradigm of a simple physical adsorption system. Noting that rare-gas atoms have closed shells (spherical symmetry), it is typically assumed that the adatom-surface interaction is nondirectional and determined by an interplay of van der Waals attraction and Pauli repulsion. ${ }^{1-3}$ Due to the simple electronic structure of rare-gas atoms and the weak interaction with surfaces, these systems have had a long tradition in serving as a model for obtaining a fundamental understanding of the process of adsorption in the field of surface science. Rare-gas atoms have also been used in different experimental techniques to probe surfaces, e.g., helium atom scattering ${ }^{4}$ (HAS) and photoemission of adsorbed $\mathrm{Xe}(\mathrm{PAX}) .^{5}$ Obviously to properly interpret experimental results it is important that the interaction of rare-gas atoms with metal surfaces is understood.

The weak interaction between rare-gas atoms and metal surfaces has often been described using pair potentials like Lennard-Jones (LJ) or Yukawa-6 (Y6) potentials. ${ }^{1,6-8}$ For the case of bulk Xe the LJ potential correctly predicts the bulk face-centered cubic (fcc) structure, over the simple cubic (sc) and body-centered cubic (bcc) structures. ${ }^{9,10}$ Because the LJ potential favors close-packed structures, this led to the expectation that rare-gas adatoms on metal surfaces preferentially bind in high coordination hollow sites. Furthermore, analogy to the behavior of rare-gas atoms on graphite, where it was found from empirical interatomic potentials that the rare-gas atoms occupy the hollow site, also led to the expectation that the most stable site on a fcc(111) metal surface would be at a threefold hollow site. ${ }^{1}$ Indeed calculations based on the $\mathrm{LJ}$ potentials found that $\mathrm{Xe}$ on $\mathrm{Ni}(110)$ binds in the hollow site. ${ }^{8}$ Furthermore, a study of $\mathrm{Xe} / \mathrm{Pt}(111)$ using an interatomic pair potential found that almost all available experimental results for this system can be reproduced by the assumption that Xe adsorbs in hollow sites. ${ }^{11}$ In fact the hollow site preference was thought to be confirmed experimen- tally by spin-polarized low-energy electron diffraction (LEED) studies ${ }^{12,13}$ of $\mathrm{Xe}$ on the $\mathrm{Pd}(111)$ and $\mathrm{Pt}(111)$ surfaces in the $(\sqrt{3} \times \sqrt{3}) R 30^{\circ}$ (from now on labeled $\sqrt{3}$ ) structure. Furthermore, recent LEED studies ${ }^{14}$ also found that Xe adatoms on graphite (0001) in the $\sqrt{3}$ structure adsorb in the hollow sites.

This widely accepted, apparent hollow site preference of $\mathrm{Xe}$ adatoms on metal surfaces was first questioned about 10 years ago when it was suggested by Gottlieb ${ }^{15}$ that Xe on $\mathrm{Pt}(111)$ might prefer low coordination sites, i.e., on-top sites, which was based on HAS data from the striped incommensurate structure (uniaxially compressed, $\Theta_{\mathrm{Xe}}<1 / 3$ ). Almost at the same time, Black and Janzen ${ }^{16}$ using interatomic potentials were not able to find a Xe-Pt pair potential which would fit the experimental observed corrugation and perpendicular vibrational frequency using the hollow site assumption for the Xe adatoms, which might indicate the preference for low coordination sites. ${ }^{1}$ Furthermore, Barker et al. ${ }^{17}$ using an empirical potential energy function to describe the interaction between $\mathrm{Xe}$ and $\mathrm{Pt}(111)$, obtained that the on-top site preference is consistent with a wide range of dynamical and equilibrium experimental data. This on-top site proposal was quite surprising, and at the time, largely not accepted by the community.

In the recent years, quantitative LEED intensity analysis apparently have confirmed the on-top adsorption site for $\mathrm{Xe}$ adatoms on $\mathrm{Cu}(111), \mathrm{Pd}(111), \mathrm{Ru}(0001)$, and $\mathrm{Pt}(111)$ in the $\sqrt{3}$ structure. ${ }^{18-21}$ A recent LEED study of $(12 \times 2)$ $-\mathrm{Xe} / \mathrm{Cu}(110)$ at $20 \mathrm{~K}$ found that rows of $\mathrm{Xe}$ atoms also adsorb on top of substrate $\mathrm{Cu}$ atoms. ${ }^{22}$ Furthemore, Seyller et al. ${ }^{23}$ claimed that rows of $\mathrm{Kr}$ adatoms are located on top of the copper substrate rows for $\mathrm{Kr} / \mathrm{Cu}(110)$ in the $c(2 \times 8)$ stuctrure at $25 \mathrm{~K}$. In addition, there is experimental evidence from scanning tunneling microscopy (STM) that Xe adatoms occupy low coordination sites on non-close-packed surfaces like $\mathrm{Ni}(110){ }^{24,25}$ Also, low-temperature STM studies found that Xe atoms adsorb in the low coordination on-top sites at the top of a step edge on $\mathrm{Pt}(111)$, rather than at the highly 
coordinated site at the bottom of the step. ${ }^{26}$ Thus, we can conclude that the experimental evidence that Xe adatoms occupy low coordination sites on non-close-packed metal surfaces is convincing.

With respect to the role of temperature in the Xe adsorption site preference, to our knowledge there has been no systematic investigations. We stress however that for Xe, the temperature and coverage $\left(\Theta_{\mathrm{Xe}}\right)$ affect the stability of the commensurate $\sqrt{3}$ structure. For example, the $\sqrt{3}$ structure appears to form only in a narrow temperature regime. HAS studies of the two-dimensional phase diagram of Xe adsorption on $\operatorname{Pt}(111)$ found that the $\sqrt{3}$ structure is stable for $\Theta_{\mathrm{Xe}} \leqslant 1 / 3$ and for surface temperatures from $62 \mathrm{~K}$ to $99 \mathrm{~K} .{ }^{27}$ Furthermore, the $\sqrt{3}$ structure for Xe on $\mathrm{Pt}(111)$ was also reported by LEED intensity analyses at temperatures of $80 \mathrm{~K}$ and $110 \mathrm{~K} .{ }^{21}$ For $\mathrm{Xe} / \mathrm{Pd}(111)$, it was observed that the $\sqrt{3}$ structure exists at $70 \mathrm{~K}$ (Ref. 12) and at $77 \mathrm{~K},{ }^{19}$ while for the case of $\mathrm{Xe}$ adsorption on $\mathrm{Cu}(111)$, the $\sqrt{3}$ structure is observed at a temperature of $50 \mathrm{~K}$ by LEED. ${ }^{18}$ These results indicate that the temperature and substrate properties, e.g., lattice constant, are important in stabilizing the $\sqrt{3}$ phase, ${ }^{28}$ however, as mentioned above, there is no evidence that temperature effects determine the adsorption site.

With regard to first-principles investigations for Xe adsorption on metals surfaces, the history in brief, is as follows. Density-functional theory (DFT) within local-density approximation (LDA) and generalized gradient approximation (GGA) have found that rare-gas adatoms polarize as it approches the metal surface, ${ }^{30-32}$ however, the role of the raregas polarization on the adsorption site preference has not been discussed. At the equilibrium configurations, DFT-LDA calculations for the $\mathrm{Xe} / \mathrm{Pt}(111)$ system using periodic ${ }^{33}(\sqrt{3}$ structure) and cluster $^{29}$ (13 Pt atoms) approaches to model the surface found that the on-top site was preferred over the hollow sites by 45 and $30 \mathrm{meV}$, respectively. Betancourt and Bird $^{33}$ also found similar conclusions using DFT-GGA, however, the on-top site is preferred over the hollow sites only by $5.0 \mathrm{meV}$. The cluster calculations can only be compared with low Xe coverage results and not the $\sqrt{3}$ phase; this could explain the difference in the relative adsorption energies results. Betancourt and Bird ${ }^{33}$ did not offer any explanation as to why $\mathrm{Xe}$ atoms adsorb in the on-top site.

The preference for low coordinated sites was also obtained for $c(2 \times 2)-\mathrm{Xe} / \mathrm{Ag}(001)$ using DFT-GGA and employing all-electron full-potential linearized plane wave (FP-LAPW) method. ${ }^{34}$ We note, however, that the $c(2 \times 2)$ $-\mathrm{Xe} / \mathrm{Ag}(001)$ structure does not occur in nature. Furthermore, the effect of spin-orbit (SO) corrections for the valence states with regard to the adsorption site and band structure were carefully discussed by Clarke et al. ${ }^{34}$ where it was found that SO corrections play an important role only for the band structure and the influence on the geometrical parameters and adsorption energies are negligible.

The nature of the interaction between $\mathrm{Xe}$ and $\mathrm{Pt}(111)$ was described in Müller's work ${ }^{29}$ as involving a hybridization of the Xe $5 p$-states with metal $d$-states, giving rise to occupied bonding and antibonding states (which contribute a repulsive interaction), and to (attractive) mixing of Xe $5 p$ states with empty metal states; the on-top site affording the greater mixing and hence being the preferred adsorption site. Because on-top adsorption was also found experimentally for Xe on $\mathrm{Cu}(111)$, for which the $d$-band lies well below the Fermi level (in our calculation the top of the $d$-band is at $\epsilon_{F}$ $-1.60 \mathrm{eV}$ ), a decisive role of the substrate $d$-states of the noble metals for the adsorption site preference has been questioned. ${ }^{18}$

Similar to Müller's suggestion, the explanation of Clarke et al. $^{34}$ is centered around the identification of occupied bonding and antibonding states involving Xe $5 p$ and metal $d$ states, leading them to the conclusion that there is a chemical component to the bonding. A chemical bond between Xe atoms and transition metals have also been suggested (based on Mulliken population analysis) for diatomic molecules like $\mathrm{XePd}$ and $\mathrm{XePt}$ using quasirelativistic pseudopotential-based quantum chemical methods. ${ }^{35}$ Although this study supports the existence of a chemical bonding, this cannot necessarily be extended to the interaction between Xe atoms and metal surfaces.

Müller also suggested that there is charge transfer from the Xe adatom to $\mathrm{Pt}(111)$ of 0.085 (on-top) and 0.075 (hollow) electrons, i.e., the Xe adatom is positively charged. ${ }^{29}$ This suggestion is in apparent agreement to experimental values of the work function change upon Xe adsorption on various metal surfaces, which show that there is a decrease in the work function, which indicates that the Xe adatom behaves as an adsorbate with an effective positive charge. However, Clarke et al. ${ }^{36,37}$ performing self-consistent electronic structure calculations using the embedding approach to include the semi-infinite substrate (treating the top two layers of atoms explicitly), found that there is an effective negative charge on the Xe atom of $-0.090|e|$ and a positive effective charge of $+0.086|e|$ on the $\mathrm{Ag}$ atom directly below $\mathrm{Xe}$, for the $c(2 \times 2)-\mathrm{Xe} / \mathrm{Ag}(001)$ system, which is in contrast to Müller's work. ${ }^{29}$

A different mechanism to the study mentioned above was suggested by Bagus et al. ${ }^{38}$ Using a 10-atom cluster model and performing Hartree-Fock calculations for $\mathrm{Xe} / \mathrm{Cu}(111)$, Bagus et al. concluded that the interface dipole moment for physisorption systems originates from "exchange-like effects," i.e., quantum effects related to the antisymmetrization of the electron wave function, and not from chemical and/or electrostatic effects. This work, however, has some limitations aside from the small cluster size used and the wellknown convergence problems of surface properties with cluster size, namely, the calculations were carried out using an effective core potential, where only the $\mathrm{Cu} 4 s$ electrons were treated explicitly. Thus, the role of the $d$-states in the interaction between $\mathrm{Xe}$ atoms and $\mathrm{Cu}$ surfaces is unclear.

We point out that as little the nature of covalent, metallic, and ionic bonding is well defined, that of van der Waals bonding is equally so. The concept of the van der Waals interaction (dispersion forces) is valid for large distances where orbitals almost do not overlap, however, at closer distances, near the equilibrium geometry of an adsorbate on a surface, the direct interaction of adsorbate and substrate orbitals is significant. In this regime the physics can be more complex: (i) the substrate electrostatic field (or the full substrate effective Kohn-Sham potential) may induce a polariza- 
tion of the adsorbate and thus induce a static dipole moment, which in turn interacts with the metal substrate electron density; (ii) the closer approach might cause wave function mixing and thus chemical interaction which may contribute to the attraction in addition to defining the repulsion. In fact the equilibrium distance is determined by a balance between the attractive forces (whatever their origin is) and Pauli repulsion. Thus, at this distance some wave function overlap does exist indeed; (iii) in the context of He- and Ne-atom-metal interactions, it was recently shown by Petersen $e t$ al. ${ }^{32}$ that the Pauli repulsion is site dependent which has noticeable effects on $\mathrm{He}$ and $\mathrm{Ne}$ scattering; (iv) the lateral interaction between the rare-gas adatoms for high coverages $\left(\Theta_{\mathrm{Xe}}\right.$ $\approx 1 / 3$ ) could conceivably play a role in the on-top site preference and in the mechanism of the interaction.

We can see from the reported experimental and theoretical results that there have been many unclear issues and controversial points concerning the interaction between $\mathrm{Xe}$ and metal surfaces. To gain a deeper insight into these systems, and to indeed determine and explain the adsorption site preference, we study the adsorption of $\mathrm{Xe}$ atoms on the $\mathrm{Mg}(0001), \mathrm{Al}(111), \mathrm{Ti}(0001), \mathrm{Cu}(111), \operatorname{Pd}(111)$, and $\mathrm{Pt}(111)$ surfaces employing DFT and the all-electron FP-LAPW method. Some of these results were briefly reported in Ref. 39. The paper is organized as follows: In Sec. II, we describe the theoretical approach and computational details. In Sec. III, we present the bulk and clean surface properties. In Sec. IV, we present results for Xe adsorption on metal surfaces for different Xe coverages, while in Sec. V, we present and discuss results for Xe/metal in the $\sqrt{3}$ structure. In Secs. VI and VII we address the concept of a chemical contribution to the binding between $\mathrm{Xe}$ atoms and metal surfaces, and the origin of the Xe adsorption site preference, respectively. The main conclusions are summarized in Sec. VIII, and in the Appendix, convergence tests for the atomic structure, work function change, and adsorption energy as a function of energy cutoff and $\mathbf{k}$-points are reported.

\section{THEORETICAL APPROACH}

All calculations are performed using DFT $^{40,41}$ with the $\mathrm{LDA}^{42}$ and $\mathrm{GGA}^{43}$ to describe the exchange-correlation energy functional. We use the recent GGA formulation of Perdew, Burke, and Ernzerhof (PBE).$^{44}$ The Kohn-Sham equations are solved using the all-electron FP-LAPW method, ${ }^{45}$ as implemented in the WIEN code. ${ }^{46-48}$ This implementation includes total energy and atomic force calculations, which allows a full structure optimization via a damped moleculardynamics approach. ${ }^{49}$ Furthermore, the spin-orbit correction for the semicore and valence states can be included by the second variational method, which is important for studying the role of spin-orbit coupling in the Xe adsorption site preference.

The LAPW wave functions in the interstitial region are represented using a plane wave expansion, truncated to include only plane waves that have kinetic energies less than some particular cutoff energy, $E^{\mathrm{wf}}$, and for the potential representation in the interstitial region, plane waves with energies up to $E^{\text {pot }}$ are considered. In the present work we used
$E^{\mathrm{wf}}=18.40 \mathrm{Ry}, 14.06 \mathrm{Ry}, 14.06 \mathrm{Ry}, 16.73 \mathrm{Ry}, 14.06 \mathrm{Ry}$, and $16.73 \mathrm{Ry}$ were used to study $\mathrm{Xe}$ on the $\mathrm{Mg}(0001)$, $\mathrm{Al}(111), \mathrm{Ti}(0001), \mathrm{Cu}(111), \mathrm{Pd}(111)$, and $\mathrm{Pt}(111)$ surfaces, respectively. $E^{\mathrm{pot}}=144 \mathrm{Ry}(\mathrm{LDA})$ and up to $256 \mathrm{Ry}(\mathrm{PBE})$ for Xe on all metal surfaces.

Inside the muffin-tin spheres with radius $R_{\mathrm{m}}$, the wave functions are expanded in radial functions (solution of the radial Schrödinger equation) times spherical harmonics up to $l_{\max }^{\mathrm{wf}}$, and for the representation of the potential inside the muffin-tin spheres, a maximum of $l_{\max }^{\mathrm{pot}}$ is used. It is important to note that the definition of the muffin-tin and interstitial regions is only used to define the augmentation for the plane waves and does not imply a shape restriction of the potential or the electron density. In the present work we used $R_{\mathrm{mt}}$ $=1.11 \AA(\mathrm{Mg}), R_{\mathrm{mt}}=1.27 \AA(\mathrm{Al}, \mathrm{Ti}, \mathrm{Pd}), R_{\mathrm{mt}}=1.16 \AA(\mathrm{Cu}$, $\mathrm{Pt}), R_{\mathrm{mt}}=1.38 \AA(\mathrm{Xe}), l_{\max }^{\mathrm{wf}}=10$, and $l_{\mathrm{max}}^{\mathrm{pot}}=4$ for all calculations.

The core states are treated fully relativistically, while the semicore and valence states are treated by the scalar relativistic approximation, i.e., the spin-orbit coupling is neglected. The linearization energies are set to be in the center of gravity of the occupied band with the respective character $(s, p$, $d, f$ ). Local orbitals (LOs) are used to describe the semicore states. Furthermore, extra LOs are added to improve the description of the valence metal $d$-states.

The clean and Xe-covered metal surfaces are modeled by the supercell approach using repeated slab geometries, which consist of several atomic layers separated by a vacuum region of finite size. In the present work we use slabs with 5 to 7 layers and a vacuum region of $18 \AA$ to $25 \AA$. The Xe atoms are adsorbed on both sides of the slab. The Xe atoms and all metal atoms in the two outermost metal layers are allowed to relax, while the central layers of the slab are kept frozen in the bulk positions. For the surface calculations, we use the theoretical equilibrium bulk lattice constants, which is critical for obtaining accurate atomic relaxations at surfaces. ${ }^{50}$

For the integration of the Brillouin zone (BZ) we use the special point method with the broadening of the Fermi surface by the Fermi distribution function with an artificial broadening parameter of $0.05 \mathrm{eV}$ in all cases. This scheme avoids the instability coming from level crossings in the vicinity of the Fermi surface in metallic systems and also reduces the number of $\mathbf{k}$-points which are necessary to perform the integration. ${ }^{45,51}$ The special $\mathbf{k}$-points are generated using a Monkhorst-Pack ${ }^{52}$ (MP) type scheme, and the total energy is extrapolated to $T=0 \mathrm{~K}$ using the correction proposed in Ref. 53. The BZ integration for the clean surface is performed using a $(16 \times 16 \times 1)$ MP grid for the $(1 \times 1)$ surface unit cell, hence, there are $30 \mathbf{k}$-points in the irreducible part of the Brillouin zone (IBZ). For Xe adsorption on the surfaces, the MP grid is chosen so as to obtain similar high quality sampling of the reciprocal space, namely a $(6 \times 6)$ mesh is used for the $(\sqrt{3} \times \sqrt{3}) R 30^{\circ}$ surface unit cell. The accuracy of the numerical approximations (k-points and energy cutoff), in relation to the calculated surface properties of Xe adsorption, are discussed in the Appendix.

\section{BULK AND CLEAN SURFACES}

We first calculate the properties of the bulk metal systems in their most stable phase, i.e., hexagonal close-packed for 
TABLE I. Bulk cohesive properties. The equilibrium lattice constant, $a_{0}$, the bulk modulus, $B_{0}$, and the cohesive energy, $E_{\text {coh }}$. The relative errors with respect to the experimental values are given in percent. For the case of bulk $\mathrm{Mg}$ and $\mathrm{Ti}$, which have the hexagonal close-packed structure, the ratio $c_{0} / a_{0}$ is 1.623 (LDA) and 1.620 (PBE) for $\mathrm{Mg}$ and 1.577 (LDA) and 1.579 (PBE) for Ti.

\begin{tabular}{|c|c|c|c|c|c|c|}
\hline \multirow{2}{*}{$\frac{\text { Bulk }}{\mathrm{Mg}}$} & \multirow[b]{2}{*}{ LDA } & \multicolumn{2}{|c|}{$a_{0}(\AA)$} & $B_{0}(\mathrm{Mbar})$ & \multicolumn{2}{|c|}{$E_{\text {coh }}(\mathrm{eV})$} \\
\hline & & 3.13 & $-2.49 \%$ & $0.38+8.57 \%$ & -1.76 & $+16.56 \%$ \\
\hline & PBE & 3.20 & $-0.31 \%$ & $0.34-2.86 \%$ & -1.50 & $-0.66 \%$ \\
\hline & Expt. $^{\mathrm{a}}$ & 3.21 & & 0.35 & -1.51 & \\
\hline \multirow[t]{3}{*}{$\mathrm{Al}$} & LDA & 3.98 & $-1.73 \%$ & $0.84+16.67 \%$ & -4.07 & $+20.06 \%$ \\
\hline & PBE & 4.04 & $-0.25 \%$ & $0.78+8.33 \%$ & -3.60 & $+6.19 \%$ \\
\hline & Expt. $^{a}$ & 4.05 & & 0.72 & -3.39 & \\
\hline \multirow[t]{3}{*}{$\mathrm{Ti}$} & LDA & 2.87 & $-2.71 \%$ & $1.26+20.00 \%$ & -6.70 & $+38.14 \%$ \\
\hline & PBE & 2.94 & $-0.34 \%$ & $1.12+6.67 \%$ & -5.87 & $+21.03 \%$ \\
\hline & Expt. $^{a}$ & 2.95 & & 1.05 & -4.85 & \\
\hline \multirow[t]{3}{*}{$\mathrm{Cu}$} & LDA & 3.52 & $-2.49 \%$ & $1.92+40.15 \%$ & -4.57 & $+30.95 \%$ \\
\hline & PBE & 3.63 & $+0.55 \%$ & $1.42+3.65 \%$ & -3.51 & $+0.57 \%$ \\
\hline & Expt. $^{a}$ & 3.61 & & 1.37 & -3.49 & \\
\hline \multirow[t]{3}{*}{$\mathrm{Pd}$} & LDA & 3.85 & $-1.03 \%$ & $2.22+22.65 \%$ & -5.04 & $+29.60 \%$ \\
\hline & PBE & 3.95 & $+1.54 \%$ & $1.63-9.94 \%$ & -3.63 & $-6.68 \%$ \\
\hline & Expt. $^{a}$ & 3.89 & & 1.81 & -3.89 & \\
\hline \multirow[t]{3}{*}{$\mathrm{Pt}$} & LDA & 3.89 & $-0.77 \%$ & $3.05+9.71 \%$ & -7.16 & $+22.60 \%$ \\
\hline & PBE & 3.97 & $+1.28 \%$ & $2.41-13.31 \%$ & -5.59 & $-4.28 \%$ \\
\hline & Expt. $^{\mathrm{a}}$ & 3.92 & & 2.78 & -5.84 & \\
\hline \multirow[t]{3}{*}{$\mathrm{Xe}$} & LDA & 5.84 & $-4.73 \%$ & 0.06 & -0.20 & $+25.00 \%$ \\
\hline & PBE & 7.05 & $+15.01 \%$ & 0.01 & -0.03 & $-81.25 \%$ \\
\hline & Expt. $^{\mathrm{a}}$ & 6.13 & & & -0.16 & \\
\hline
\end{tabular}

Reference 10.

$\mathrm{Mg}$ and $\mathrm{Ti}$, and face-centered cubic for $\mathrm{Al}, \mathrm{Cu}, \mathrm{Pd}$, and $\mathrm{Pt}^{9}{ }^{9,10}$ We also calculate the bulk Xe properties in the face-centered cubic structure. Table I shows the equilibrium lattice constants, bulk moduli, and cohesive energies where we used Murnaghan's equation of state ${ }^{54}$ to determine the two former quantities. Calculation of the cohesive energy requires the total energy of the free atom. For this, we performed spinpolarized calculations using a cubic box with side length $10.58 \AA$ and one k-point in the IBZ, i.e., $\left(\frac{1}{4}, \frac{1}{4}, \frac{1}{4}\right)(2 \pi / a)$. In this way the total energy of the bulk and free atom systems are calculated using the same type and size of basis set; e.g., at the scalar relativistic level for the valence states and inclusion of spin-orbit coupling only for the core states. We found in earlier work ${ }^{55}$ that the chosen $\mathbf{k}$-point yielded faster convergence of the total energy with cell size as compared to selecting the $\Gamma$-point. In calculating the cohesive energies, no corrections are made for the zero point energy. For the bulk metals, on the whole, the PBE yields a better overall agreement with experimental results, while the LDA provides a better description of the equilibrium lattice constant for bulk Xe. Further details and comparisons can be found in Ref. 50.

With respect to the clean surface properties, we calculate the work function, $\Phi$, surface energy, $\sigma$, and interlayer relaxations, $\Delta d_{i, i+1}$, with respect to the bulk interlayer spacing. Convergence tests for the clean $\mathrm{Cu}(111)$ surface properties with respect to the number of layers in the slab are reported in Ref. 50. Only $\Phi$ and $\Delta d_{12}$ are listed in Table II, which are relevant for the present paper. The agreement between theory and available experimental results for the interlayer relaxation and work function is generally good, but there are some deviations. For $\Delta d_{12}$, the LDA and PBE predict values close to the LEED results ${ }^{56,57}$ for $\mathrm{Mg}(0001), \mathrm{Al}(111), \mathrm{Cu}(111)$, and $\mathrm{Pt}(111)$, however for $\mathrm{Ti}(0001)$ the LDA and PBE overestimate the interlayer relaxation by a factor of 3 . For $\operatorname{Pd}(111)$, the calculated work functions are significantly smaller than experiment; in this case we question the accuracy of the experimental value. Other surface properties and further details and comparison with other first-principles results can be found in Ref. 50.

\section{Xe ADSORPTION ON METAL SURFACES}

To determine the energetically favorable adsorption site for $\mathrm{Xe}$ on $\mathrm{Mg}(0001), \mathrm{Al}(111), \mathrm{Ti}(0001), \mathrm{Cu}(111), \mathrm{Pd}(111)$, and $\operatorname{Pt}(111)$, in the $\sqrt{3}$ structure, as well as to provide an improved understanding of the interaction mechanism between rare-gas atoms and metal surfaces, we choose six adsorption sites on the hcp(0001) and fcc(111) surfaces, namely, on-top, top-fcc (midpoint between the on-top and fcc sites), fcc, bridge, hcp, and top-hcp (midpoint between the on-top and hep sites) sites, which are indicated in Fig. 1. For fcc(111), the two threefold sites, i.e., the fcc and hcp sites, differ only in that the fcc site has a substrate atom directly under it in the third layer, while the hcp site has a substrate atom directly under it in the second layer. For hcp(0001), the 
TABLE II. Relaxation of the first interlayer spacing, $\Delta d_{12}$, and the work function, $\Phi$, of the clean close-packed surfaces.

\begin{tabular}{|c|c|c|c|c|c|c|}
\hline \multirow[b]{2}{*}{ Surface } & \multicolumn{3}{|c|}{$\Delta d_{12}(\%)$} & \multicolumn{3}{|c|}{$\Phi(\mathrm{eV})$} \\
\hline & LDA & PBE & Expt. & LDA & PBE & Expt. \\
\hline $\operatorname{Mg}(0001)$ & +1.41 & +1.32 & $+1.76^{\mathrm{a}}$ & 3.83 & 3.69 & \\
\hline $\mathrm{Al}(111)$ & +1.35 & +1.35 & $+1.30^{\mathrm{b}}$ & 4.21 & 4.04 & $4.30^{\mathrm{g}}$ \\
\hline $\mathrm{Ti}(0001)$ & -6.37 & -6.64 & $-2.10^{\mathrm{c}}$ & 4.66 & 4.40 & \\
\hline $\mathrm{Cu}(111)$ & -1.25 & -1.19 & $-0.70^{\mathrm{d}}$ & 5.22 & 4.78 & $4.94^{\mathrm{h}}$ \\
\hline $\operatorname{Pd}(111)$ & -0.22 & -0.01 & $+2.40^{\mathrm{e}}$ & 5.64 & 5.22 & $5.95^{\mathrm{i}}$ \\
\hline $\operatorname{Pt}(111)$ & +0.88 & +1.14 & $+1.09^{\mathrm{f}}$ & 6.06 & 5.69 & $5.93^{\mathrm{g}}$ \\
\hline
\end{tabular}

${ }^{\mathrm{a}}$ Reference 60 .

${ }^{\mathrm{b}}$ Reference 61 .

${ }^{c}$ Reference 58.

${ }^{\mathrm{d}}$ Reference 56.

${ }^{\mathrm{e}}$ Reference 59.

${ }^{\mathrm{f}}$ Reference 57.

geference 62.

heference 63.

${ }^{i}$ Reference 64.

situation is the same except that the fcc site has no substrate atom directly under it at all (see Fig. 1).

We first explore the effect of lateral Xe interactions on the adsorption site preference by performing calculations for different coverages, i.e., $\Theta_{\mathrm{Xe}}=1 / 3(\sqrt{3}$ unit cell), $1 / 4[(2 \times 2)$ unit cell], and $1 / 9[(3 \times 3)$ unit cell $]$ for adsorption in the on-top and threefold fcc sites on the $\operatorname{Pd}(111)$ and $\operatorname{Pt}(111)$ surfaces. The adsorption energy is given by

$$
E_{\mathrm{ad}}\left(\Theta_{\mathrm{Xe}}\right)=\frac{1}{2}\left[E_{\text {tot }}^{\mathrm{Xe}}{ }^{\mathrm{metal}}\left(\Theta_{\mathrm{Xe}}\right)-E_{\text {tot }}^{\text {metal }}-2 E_{\text {tot }}^{\mathrm{Xe}}\right],
$$

where $E_{\text {tot }}^{\mathrm{Xe} / \mathrm{metal}}\left(\Theta_{\mathrm{Xe}}\right), E_{\text {tot }}^{\text {metal }}$, and $E_{\text {tot }}^{\mathrm{Xe}}$ are the total energy of the $\mathrm{Xe}$ and/or metal system for a given Xe coverage, the clean metal surface, and the free Xe atom, respectively. The factors 2 and $1 / 2$ are due to the adsorption of Xe atoms on both sides of the slab. The adsorption energies as function of the Xe coverage are summarized in Table III.

Thus, the on-top site is favored for Xe coverages of $1 / 3$, $1 / 4$, and $1 / 9$ for $\mathrm{Xe}$ adatoms on $\mathrm{Pd}(111)$ and $\mathrm{Pt}(111)$. For $\mathrm{Xe} / \mathrm{Pt}(111)$, with decreasing coverage the difference between on-top and fcc sites increases, whereas for $\mathrm{Xe} / \mathrm{Pd}(111)$, this difference decreases with decreasing $\mathrm{Xe}$ coverage. The results show clearly that the lateral interactions between the Xe adatoms do not play a critical role in the Xe adsorption site preference. Therefore, we can conclude that the preferred Xe adsorption site is determined by the interaction between adsorbate and substrate and hence, the study of Xe at just $\Theta_{\mathrm{Xe}}=1 / 3$ can be used to understand and explain the adsorption site preference. From the results reported in Table III it can be seen that for low Xe coverages there is an effective repulsive interaction between $\mathrm{Xe}$ atoms as the adsorption energy becomes less favorable when increasing the coverage from $1 / 9$ to $1 / 4$. For higher coverages the nature of this interaction changes to attractive, however, towards formation of the $\sqrt{3}$ structure.

We also investigate the effect of spin-orbit (SO) corrections for the $\mathrm{Xe}$ and metal valence states of the $\mathrm{Xe} / \mathrm{Pd}(111)$ and $\mathrm{Xe} / \mathrm{Pt}(111)$ systems on the preferred adsorption site. We find that $\Delta E_{\mathrm{ad}}^{\mathrm{ft}}$ is only slightly changed $(\approx 2 \mathrm{meV})$ by the $\mathrm{SO}$ correction, and the structural parameters do not change. However, we find that the SO correction is essential to describe the correct splitting of Xe $5 p$-states into $5 p_{1 / 2^{-}}$and $5 p_{3 / 2}$-states. Thus, it is important for obtaining a correct description of the Xe adlayer band structure. Our conclusions are in agreement with the recent study reported by Clarke et $a l .{ }^{34}$ for $\mathrm{Xe} / \mathrm{Ag}(001)$ where SO corrections were also investigated as mentioned earlier.

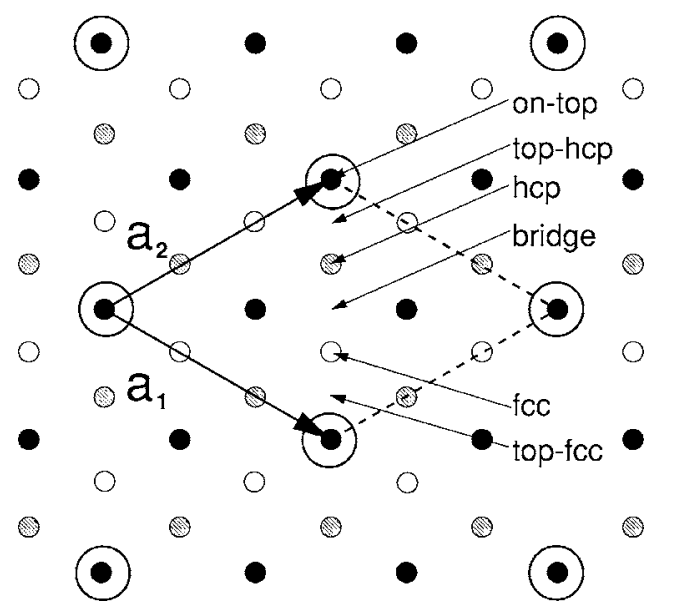

FIG. 1. Adsorption sites for Xe adatoms on the fcc(111) surface in the $\sqrt{3}$ structure. The indicated adsorption sites are on-top, tophcp, hcp, bridge, fcc, and top-fcc. The Xe adatoms and metal atoms in the topmost surface layer are indicated by large open circles and small black circles, respectively, while the second and third layer metal atoms are depicted as small grey and open circles, respectively. The Xe adatoms are placed for example in the on-top sites in the figure. For the hcp(0001) surface, the only difference is that the small white circles would sit directly below the black ones and would not be visible. 
TABLE III. Adsorption energy in $\mathrm{meV}, E_{\text {ad }}$, of Xe adatoms in the on-top sites on $\mathrm{Pd}(111)$ and $\mathrm{Pt}(111)$ as function of the Xe coverage, $\Theta_{\mathrm{Xe}}$, calculated with the LDA functional. $\Delta E_{\mathrm{ad}}^{\mathrm{ft}}$ indicate the relative adsorption energy difference in $\mathrm{meV}$ between the fcc and on-top sites $\left(\Delta E_{\mathrm{ad}}^{\mathrm{ft}}=E_{\mathrm{ad}}^{\mathrm{fcc}}-E_{\mathrm{ad}}^{\mathrm{on}-\mathrm{top}}\right)$.

\begin{tabular}{llllll}
\hline \hline & \multicolumn{3}{c}{$\operatorname{Pd}(111)$} & & \multicolumn{3}{c}{$\operatorname{Pt}(111)$} \\
\cline { 2 - 3 } \cline { 5 - 6 }$\Theta_{\mathrm{Xe}}$ & $E_{\mathrm{ad}}$ & $\Delta E_{\mathrm{ad}}^{\mathrm{ft}}$ & & $E_{\mathrm{ad}}$ & $\Delta E_{\mathrm{ad}}^{\mathrm{ft}}$ \\
\hline $1 / 3$ & -453 & 51.22 & & -353 & 49.87 \\
$1 / 4$ & -442 & 43.06 & & -341 & 53.10 \\
$1 / 9$ & -457 & 20.59 & & -365 & 57.87 \\
\hline \hline
\end{tabular}

\section{Xe ADSORPTION ON METAL SURFACES IN THE $\sqrt{3}$ STRUCTURE}

Our main focus will be on the adsorption of Xe atoms on metal surfaces in the $\sqrt{3}$ structure since the lateral interactions between the Xe adatoms do not play a critical role in the site preference. Furthermore, the $\sqrt{3}$ structure has been observed and analyzed by quantitatively LEED intensity analysis, ${ }^{18-21}$ so that our atomic geometry can be quantitatively compared to experimental results. SO corrections for the valence states will not be discussed from this point onwards, since they do not affect the adsorption energies or atomic structure.

\section{A. Lateral PES and work function change}

Figure 2 shows the relative adsorption energy with respect to the on-top site, $\Delta E_{\text {ad }}=E_{\text {ad }}^{\text {site }}-E_{\text {ad }}^{\text {on-top }}$, and the induced work function change with respect to the clean surface, $\Delta \Phi$ $=\Phi^{\mathrm{Xe} / \mathrm{metal}}-\Phi^{\mathrm{metal}}$, for $\mathrm{Xe}$ adatoms on the $\operatorname{Pd}(111)$ and $\operatorname{Pt}(111)$ surfaces in the $\sqrt{3}$ structure, obtained using the LDA functional for the mentioned six adsorption sites (see Fig. 1). Results obtained using the PBE functional are very similar, but of a smaller magnitude (see Ref. 50). The values of $E_{\mathrm{ad}}$, $\Delta E_{\mathrm{ad}}^{\mathrm{ft}}$, and $\Delta \Phi$ for all studied systems are summarized in Table IV. For the $\mathrm{Xe} / \mathrm{Mg}(0001)$ and $\mathrm{Xe} / \mathrm{Al}(111)$ systems, only LDA calculations are performed, since calculations using LDA and PBE for Xe adatoms on transition metals give the same physical picture, as will be seen below.

Table IV show clearly that Xe preferentially binds to the low coordination adsorption sites, i.e., the on-top site in all studied cases. Thus, our results are in agreement with the quantitative LEED intensity analyses performed for the $\mathrm{Xe} / \mathrm{Cu}(111),{ }^{18} \mathrm{Xe} / \mathrm{Pd}(111),{ }^{19}$ and $\mathrm{Xe} / \mathrm{Pt}(111)$ systems. $^{21}$ Our results indicate that the on-top site preference may be the rule rather than the exception for Xe adsorption on metal surfaces.

For the systems where there are available experimental results with which to compare, e.g., $\mathrm{Xe} / \mathrm{Cu}(111)$, $\mathrm{Xe} / \mathrm{Pd}(111)$, and $\mathrm{Xe} / \mathrm{Pt}(111)$, it can be seen from Table IV that the adsorption energies calculated using LDA are closer to the experimental results than those obtained with the PBE functional. Basically, the adsorption energies calculated by PBE are smaller than the LDA values, which follows the same trend found for the cohesive energy of bulk Xe. For the case where there are other theoretical calculations, i.e.,
$\mathrm{Xe} / \mathrm{Pt}(111)$, our results are in relatively good agreement with them (see Table IV).

It can be seen from Table IV that the adsorption energy is largest for the "true" transition metals, namely, $\operatorname{Pd}(111)$ and $\mathrm{Pt}(111)$, while Xe adsorption on the free-electron-like metal surfaces has a significantly smaller adsorption energy. That of $\mathrm{Xe}$ on $\mathrm{Cu}(111)$ lies in between. For $\mathrm{Xe}$ on $\mathrm{Ti}(0001)$ the adsorption energy is similar to the free-electron-like metal substrates. The following trend in adsorption energy can be noticed: $\quad E_{\mathrm{ad}}^{\mathrm{Xe} / \mathrm{Mg}(0001)}<E_{\mathrm{ad}}^{\mathrm{Xe} / \mathrm{Al}(111)}<E_{\mathrm{ad}}^{\mathrm{Xe} / \mathrm{Ti}(0001)}<E_{\mathrm{ad}}^{\mathrm{Xe} / \mathrm{Cu}(111)}$ $<E_{\mathrm{ad}}^{\mathrm{Xe} / \mathrm{Pt}(111)}<E_{\mathrm{ad}}^{\mathrm{Xe} / \mathrm{Pd}(111)}$, which is in good agreement with available experimental results. As we will see in the next section, the adsorption energies correlate with the Xe-metal
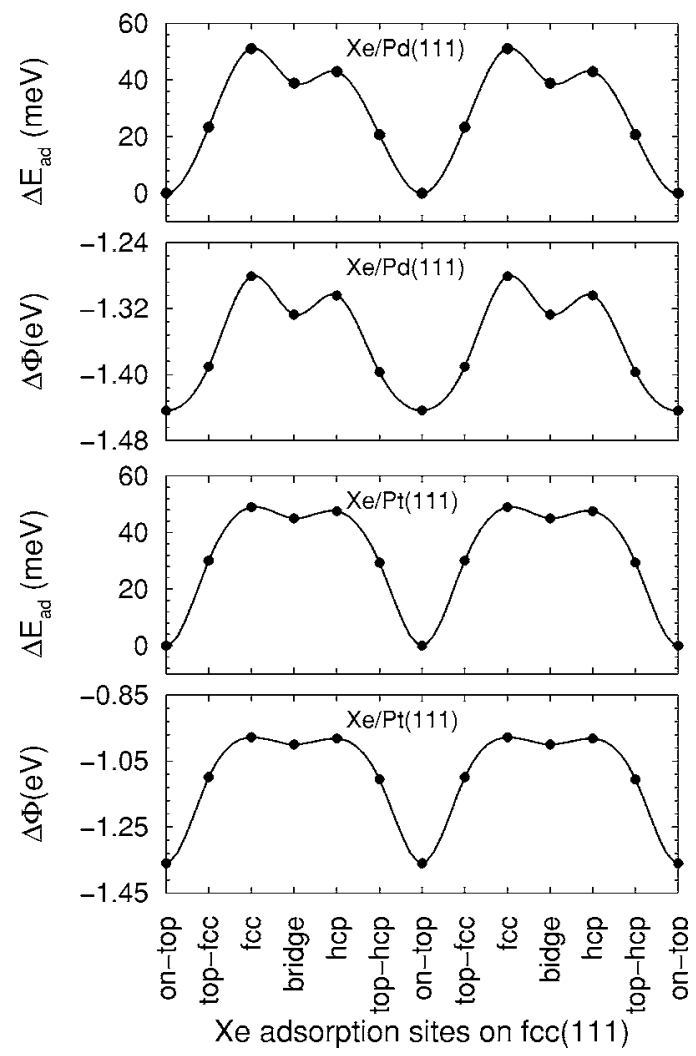

FIG. 2. DFT-LDA results for the relative adsorption energy, $\Delta E_{\mathrm{ad}}=E_{\mathrm{ad}}^{\text {site }}-E_{\mathrm{ad}}^{\text {on-top }}$, i.e., the energy of the on-top site is the energy zero, and work function change with respect to the clean surface, $\Delta \Phi=\Phi^{\mathrm{Xe} / \text { metal }}-\Phi^{\text {metal }}$, for $\mathrm{Xe}$ on the $\operatorname{Pd}(111)$ and $\operatorname{Pt}(111)$ surfaces in the $\sqrt{3}$ structure. 
TABLE IV. Adsorption properties of Xe adatoms on metal surfaces in the $\sqrt{3}$ structure. The adsorption energy, $E_{\text {ad }}$, for Xe in the on-top sites, relative adsorption energy between fcc and on-top sites, $\Delta E_{\mathrm{ad}}^{\mathrm{ft}}=E_{\mathrm{ad}}^{\mathrm{fcc}}-E_{\mathrm{ad}}^{\mathrm{on}-t o p}$, and the induced work function change upon Xe adsorption with respect to the clean surface, $\Delta \Phi=\Phi^{\mathrm{Xe} / \mathrm{metal}}-\Phi^{\mathrm{metal}}$. Experimental and other theoretical results are listed.

\begin{tabular}{|c|c|c|c|c|c|}
\hline \multirow[b]{2}{*}{ Xe/metal } & & \multirow{2}{*}{$\begin{array}{c}E_{\mathrm{ad}} \\
(\mathrm{meV})\end{array}$} & \multirow{2}{*}{$\begin{array}{l}\Delta E_{\mathrm{ad}}^{\mathrm{ft}} \\
(\mathrm{meV})\end{array}$} & \multicolumn{2}{|c|}{$\Delta \Phi(\mathrm{eV})$} \\
\hline & & & & on-top & fcc \\
\hline $\operatorname{Mg}(0001)$ & $\mathrm{LDA}^{\mathrm{a}}$ & -130 & 4.34 & -0.22 & -0.16 \\
\hline \multirow[t]{2}{*}{$\mathrm{Al}(111)$} & $\mathrm{LDA}^{\mathrm{a}}$ & -176 & 1.12 & -0.38 & -0.33 \\
\hline & Exp. & & & \multicolumn{2}{|c|}{$-0.29^{b}$} \\
\hline \multirow[t]{2}{*}{$\operatorname{Ti}(0001)$} & $\mathrm{LDA}^{\mathrm{a}}$ & -196 & 21.82 & -0.87 & -0.70 \\
\hline & $\mathrm{PBE}^{\mathrm{a}}$ & -44 & 3.10 & -0.34 & -0.28 \\
\hline \multirow[t]{3}{*}{$\mathrm{Cu}(111)$} & $\mathrm{LDA}^{\mathrm{a}}$ & -277 & 9.14 & -0.96 & -0.83 \\
\hline & $\mathrm{PBE}^{\mathrm{a}}$ & -40 & 5.91 & -0.20 & -0.20 \\
\hline & Exp. & $-183^{\mathrm{c}},-190^{\mathrm{d}}$ & & \multicolumn{2}{|c|}{$-0.48^{\mathrm{b}},-0.58^{\mathrm{b}}$} \\
\hline \multirow[t]{3}{*}{$\operatorname{Pd}(111)$} & $\mathrm{LDA}^{\mathrm{a}}$ & -453 & 51.22 & -1.44 & -1.28 \\
\hline & $\mathrm{PBE}^{\mathrm{a}}$ & -76 & 13.48 & -0.81 & -0.61 \\
\hline & Exp. & $-356^{\mathrm{c}},-360^{\mathrm{e}}$ & & \multicolumn{2}{|c|}{$-0.60^{\mathrm{b}},-0.85^{\mathrm{e}}$} \\
\hline \multirow[t]{6}{*}{$\operatorname{Pt}(111)$} & $\mathrm{LDA}^{\mathrm{a}}$ & -367 & 49.09 & -1.36 & -0.98 \\
\hline & $\mathrm{PBE}^{\mathrm{a}}$ & -82 & 4.80 & -0.58 & -0.29 \\
\hline & $\operatorname{LDA}^{\mathrm{f}}$ & -307 & 30.00 & & \\
\hline & LDA $^{g}$ & -332 & 45.00 & -1.30 & \\
\hline & $\mathrm{PBE}^{\mathrm{g}}$ & -41 & 5.00 & & \\
\hline & Exp. & $-310^{\mathrm{b}}$ & & \multicolumn{2}{|c|}{$-0.29^{b}$} \\
\hline
\end{tabular}

Present work.

${ }^{\mathrm{b}}$ Reference 62.

${ }^{\mathrm{c}}$ Reference 3.

${ }^{\mathrm{d}}$ Reference 18 .

${ }^{\mathrm{e}}$ Reference 64.

${ }^{\text {fReference } 29 .}$

gReference 33.

distance, with the strongest binding having the smallest distance.

The energy difference between the hollow and on-top sites, $\Delta E_{\mathrm{ad}}^{\mathrm{ft}}$, is larger for the transition metal surfaces than for the free-electron-like metal surfaces. It can be also noticed in Table IV that the PBE functional yields a smaller $\Delta E_{\mathrm{ad}}^{\mathrm{ft}}$ compared with LDA values. The diffusion of an Xe atom from an on-top site to a neighboring on-top site will proceed via the lowest energy pathway. It can be seen from Fig. 2 that for Xe on $\operatorname{Pt}(111)$ and $\operatorname{Pd}(111)$, this will be via the bridge site, which is slightly energetically more favorable than the hollow sites. For $\mathrm{Xe} / \mathrm{Pt}(111)$, the energy difference between on-top and bridge is $40.4 \mathrm{meV}$, which can approximately be compared with experimental results. Kern et al. ${ }^{27}$ estimate that the diffusion barrier of $\mathrm{Xe}$ on $\mathrm{Pt}(111)$ is $30 \mathrm{meV}$, while $31 \mathrm{meV}$ was reported in Ref. 65 which is in quite good agreement with the present calculations. However, a recent quasielastic helium atom scattering study at low Xe coverage on $\mathrm{Pt}(111)$, suggested that the diffusion barrier is $9.60 \mathrm{meV} .^{66}$

It can be seen from Fig. 2 that the relative adsorption energy and the induced work function change exhibit surprisingly similar behavior, that is, the maximum (minimum) change in the substrate work function occurs for Xe adatoms in the on-top (fcc) sites. We will return to this point below.
The same trend was found for all studied systems and functionals, as can be noted from Table IV. The large difference between the induced work function change obtained using the PBE compared with the LDA values is explained in terms of the difference in the equilibrium vertical distance. Calculations using the same vertical distance (3.10 $\AA$ ) for Xe adatoms above the $\mathrm{Pt}(111)$ surface and employing the LDA and $\mathrm{PBE}$, predict almost the same change in the substrate work function, i.e., $-1.29 \mathrm{eV}$ (LDA) and $-1.20 \mathrm{eV}$ (PBE). Therefore, to perform a quantitative comparison between theoretical values of the induced work function change and experimental ones, it is necessary to improve the description of the equilibrium vertical distance (see below).

\section{B. Atomic geometry}

The equilibrium structural parameters defined in Fig. 3 are summarized in Table $\mathrm{V}$ along with earlier published results. We found that the LDA (PBE) equilibrium vertical distance is about $10 \%$ shorter (larger) than those obtained by quantitative LEED intensity analyses, ${ }^{18,19,21}$ but in view of the fact that the adsorption energy is weak in physisorption systems and our theory does not include vibrational contributions, the sign and size of the deviation is as expected. It 


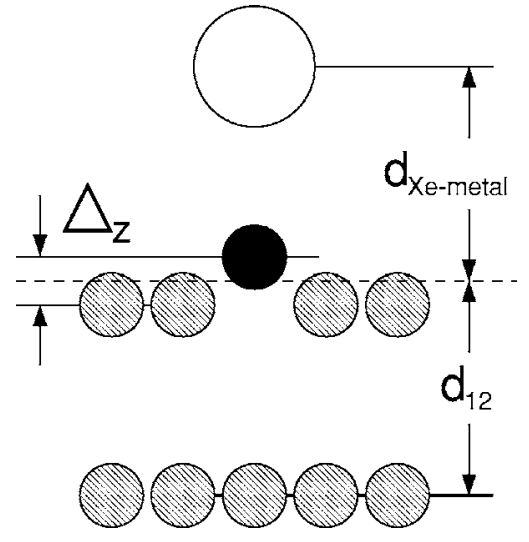

FIG. 3. Schematic diagram showing the equilibrium vertical distance between the Xe adatom and the substrate with respect to the topmost layer, $d_{\text {Xe-metal }}$, substrate rumpling, $\Delta_{z}$, and the first interlayer atomic distance, $d_{12}$. The position of the dashed line is given by the average of the upper and lower vertical distances of the atoms in the top layer. The large open circle, shaded circles, and black circle indicate the Xe adatom, metal atoms not under the Xe atom, and metal atom under the $\mathrm{Xe}$ atom, respectively.

can be noted that the equilibrium vertical distance increases as the adsorption energy decreases, i.e., $d_{\mathrm{Xe}-\text { metal }}^{\mathrm{Mg}}>d_{\mathrm{Xe}-\mathrm{Al} \text { tal }}^{\mathrm{Al}}$ $>d_{\text {Xe-metal }}^{\mathrm{Ti}}>d_{\text {Xe-metal }}^{\mathrm{Cu}}>d_{\text {Xe-metal }}^{\mathrm{Pt}}>d_{\text {Xe-metal }}^{\mathrm{Pd}}$ in perfect (inverse) sequence with the trend in adsorption energy.

There is one deviation between our calculations and the quantitative LEED intensity analyse ${ }^{18,21}$ and that is in the direction of the displacement of the metal atom below the $\mathrm{Xe}$ adatom in the on-top site. It was reported by Seyller et al. that the substrate rumpling is negative for Xe adatoms on $\mathrm{Cu}(111)$ and $\mathrm{Pt}(111)$, i.e., the metal atom under the Xe adatom moves inward, while the metal atoms not under the Xe adatom move outward, however, we find the opposite. This result was obtained using the LDA and PBE functionals. It is important to mention, however, that the uncertainties in the substrate rumpling obtained in the LEED studies ${ }^{21}$ are larger than the value itself. For example, at a temperature of $80 \mathrm{~K}$ $(110 \mathrm{~K})$, the substrate rumpling is $0.00 \AA(-0.01 \AA)$, while the error is $\pm 0.02 \AA( \pm 0.03 \AA)$. These difficulties in the quantitative LEED intensity analysis highlight the value of first-principles calculations to help in determining the correct structure and trends.

To gain insight into the possible role of substrate rumpling in determining the Xe adsorption site preference, we perform calculations with the atoms of the substrate fixed at their ideal clean surface positions. We find that this does not affect the site preference, i.e., Xe still prefers the on-top sites. The interlayer relaxations of the $\mathrm{Xe}$ and/or metal systems are

TABLE V. Structural geometry of Xe adsorption on metal surfaces in the $\sqrt{3}$ structure. Equilibrium vertical distance between the Xe adatoms and surface with respect to the topmost substrate layer, $d_{\text {Xe-metal }}$. Topmost interlayer spacing relaxation with respect to the ideal interlayer surface spacing, $\Delta d_{12}=100\left(d_{12}-d_{0}\right) / d_{0}$, and the substrate rumpling, $\Delta_{z}$. The positive (negative) sign for the substrate rumpling indicates an outward (inward) displacement of the metal atom under the Xe adatom. $d_{0}=a_{0} / \sqrt{3}$ for fcc $(111)$ and $c_{0} / 2$ for hcp $(0001)$. For further details, see Figs. 1 and 3.

\begin{tabular}{|c|c|c|c|c|c|c|}
\hline \multirow[b]{2}{*}{$\mathrm{Xe} / \mathrm{metal}$} & & \multicolumn{2}{|c|}{$d_{\text {Xe-metal }}(\AA)$} & \multicolumn{2}{|c|}{$\Delta d_{12}(\%)$} & \multirow{2}{*}{$\begin{array}{l}\Delta_{z}(\AA) \\
\text { on-top }\end{array}$} \\
\hline & & on-top & fcc & on-top & fcc & \\
\hline $\operatorname{Mg}(0001)$ & $\mathrm{LDA}^{\mathrm{a}}$ & 3.80 & 3.84 & +1.78 & +2.10 & 0.00 \\
\hline $\operatorname{Al}(111)$ & $\mathrm{LDA}^{\mathrm{a}}$ & 3.69 & 3.72 & +1.20 & +1.24 & -0.01 \\
\hline \multirow[t]{2}{*}{$\mathrm{Ti}(0001)$} & $\mathrm{LDA}^{\mathrm{a}}$ & 3.56 & 3.66 & -6.00 & -6.49 & +0.06 \\
\hline & $\mathrm{PBE}^{\mathrm{a}}$ & 4.18 & 4.27 & -6.77 & -6.82 & +0.01 \\
\hline \multirow[t]{3}{*}{$\mathrm{Cu}(111)$} & $\mathrm{LDA}^{\mathrm{a}}$ & 3.26 & 3.30 & -0.81 & -1.18 & +0.03 \\
\hline & $\mathrm{PBE}^{\mathrm{a}}$ & 4.03 & 4.14 & -1.01 & -0.95 & 0.00 \\
\hline & LEED $^{\mathrm{b}}$ & 3.60 & & -0.48 & & -0.01 \\
\hline \multirow[t]{3}{*}{$\operatorname{Pd}(111)$} & $\mathrm{LDA}^{\mathrm{a}}$ & 2.85 & 2.86 & +0.51 & +0.10 & +0.05 \\
\hline & $\mathrm{PBE}^{\mathrm{a}}$ & 3.27 & 3.41 & +0.13 & -0.15 & +0.03 \\
\hline & $\mathrm{LEED}^{\mathrm{c}}$ & 3.07 & & & & -0.02 \\
\hline \multirow[t]{6}{*}{$\operatorname{Pt}(111)$} & $\mathrm{LDA}^{\mathrm{a}}$ & 3.07 & 3.19 & +1.75 & +1.28 & +0.04 \\
\hline & $\mathrm{PBE}^{\mathrm{a}}$ & 3.62 & 3.91 & +1.43 & +1.32 & +0.02 \\
\hline & $\mathrm{LDA}^{\mathrm{d}}$ & 3.00 & 2.95 & & & \\
\hline & $\mathrm{LDA}^{\mathrm{e}}$ & 3.11 & & & & \\
\hline & $\mathrm{PBE}^{\mathrm{e}}$ & 3.80 & & & & \\
\hline & $\mathrm{LEED}^{\mathrm{f}}$ & 3.40 & & +1.76 & & 0.00 \\
\hline
\end{tabular}

apresent work.

${ }^{\mathrm{b}}$ Reference 18 .

${ }^{\mathrm{c}}$ Reference 19 .

${ }^{\mathrm{d}}$ Reference 29.

eReference 33 .

${ }^{\text {fReference } 21 .}$ 
very close to those of the clean surfaces, which is understandable because of the weak interaction between Xe atoms and metal surfaces. Interestingly the vertical distance between $\mathrm{Xe}$ and the topmost metal layer is smaller for Xe in the on-top site as compared to the fcc site, i.e., $d_{\text {Xe-metal }}^{\text {on-top }}$ $<d_{\text {Xe-metal. }}^{\text {fcc }}$. This is the case for all studied systems. This is in contrast to the first-principles calculations reported in Ref. 29. It is also in contrast to what might intuitively be expected, since the picture based on the LJ pair potential calculations ${ }^{8}$ suggests the opposite behavior, i.e., that Xe adatoms get closer to the surface in the hollow sites. Barker et al. ${ }^{17}$ however determined an empirical potential energy function for the $\mathrm{Xe} / \mathrm{Pt}(111)$ system which gives rise to a smaller equilibrium vertical distance for $\mathrm{Xe}$ in the on-top site, which is in agreement with our results.

This unexpected result for $d_{\text {Xe-metal }}$ was also found in DFT calculations ${ }^{67}$ for $\operatorname{Ar} / \operatorname{Ag}(111)$. Furthermore, in a recent work, $\mathrm{Bruch}^{68}$ represented the $\mathrm{Ar}-\mathrm{Ag}(111)$ potential energy surface by a truncated Fourier series using the first shell of reciprocal vectors. From the analysis of the coefficients in the Fourier expansion, together with the DFT calculations reported in Ref. 67, it was concluded that the equilibrium distance for $\mathrm{Ar}$ in the on-top is smaller than for the hollow site. Therefore, this behavior for $\operatorname{Ar} / \operatorname{Ag}(111)$ is the same as that for the Xe and/or metal systems. We verified carefully that this result is not an effect of unconverged calculations, as can be seen in the tests reported in the Appendix.

\section{Perpendicular PES}

In order to understand the interaction mechanism between Xe atoms and metal surfaces, and to explain the on-top site preference, we calculate the perpendicular potential energy surface (PES) for Xe in the on-top and fcc sites. The PES is obtained by total energy calculations for different Xe adatom heights, $Z_{\mathrm{Xe}}$, measured with respect to the topmost surface layer. As was discussed before, the substrate relaxation does not play a critical role for the Xe adsorption site preference, thus we keep the substrate atoms frozen in the ideal clean surface positions. The PES derived from DFT calculations is plotted with respect to the total energy at the greatest Xe adatom height above the metal surface, $Z_{\mathrm{Xe}}^{\max }$, i.e., PES $=E_{\mathrm{tot}}\left(Z_{\mathrm{Xe}}\right)-E_{\mathrm{tot}}\left(Z_{\mathrm{Xe}}^{\max }\right)$. For the LDA and PBE functionals, $Z_{\mathrm{Xe}}^{\max }=5.48$ and $6.02 \AA$, respectively. The PESs for Xe on $\operatorname{Pd}(111)$ and $\mathrm{Pt}(111)$ obtained using the LDA functional are plotted in Fig. 4.

The on-top site is the energetically most favorable site for all Xe distances above the equilibrium position for both systems. The same trend is obtained for the other systems and also for the PBE functional (see Ref. 50). However, it should be noted that for distances smaller than the equilibrium distance, the fcc site becomes energetically favorable for $\mathrm{Xe} / \mathrm{Pd}(111)$. Similar trend can be seen for $\mathrm{Xe} / \mathrm{Pd}(111)$, but for distances very close to the surface [see Fig. 4(a)]. For large distances above the surface, the PES for Xe in the on-top and fcc sites have almost the same value. For example, $\Delta E_{\text {ad }}^{\mathrm{ft}}$ for $\mathrm{Xe}$ at $Z_{\mathrm{Xe}}=5.48 \AA$ above the $\operatorname{Pt}(111)$ surface is smaller than $0.40 \mathrm{meV}$ employing LDA, while for $\mathrm{Xe}$ at $Z_{\mathrm{Xe}}=6.02 \AA$ and employing the PBE, $\Delta E_{\mathrm{ad}}^{\mathrm{ft}}$ is smaller than
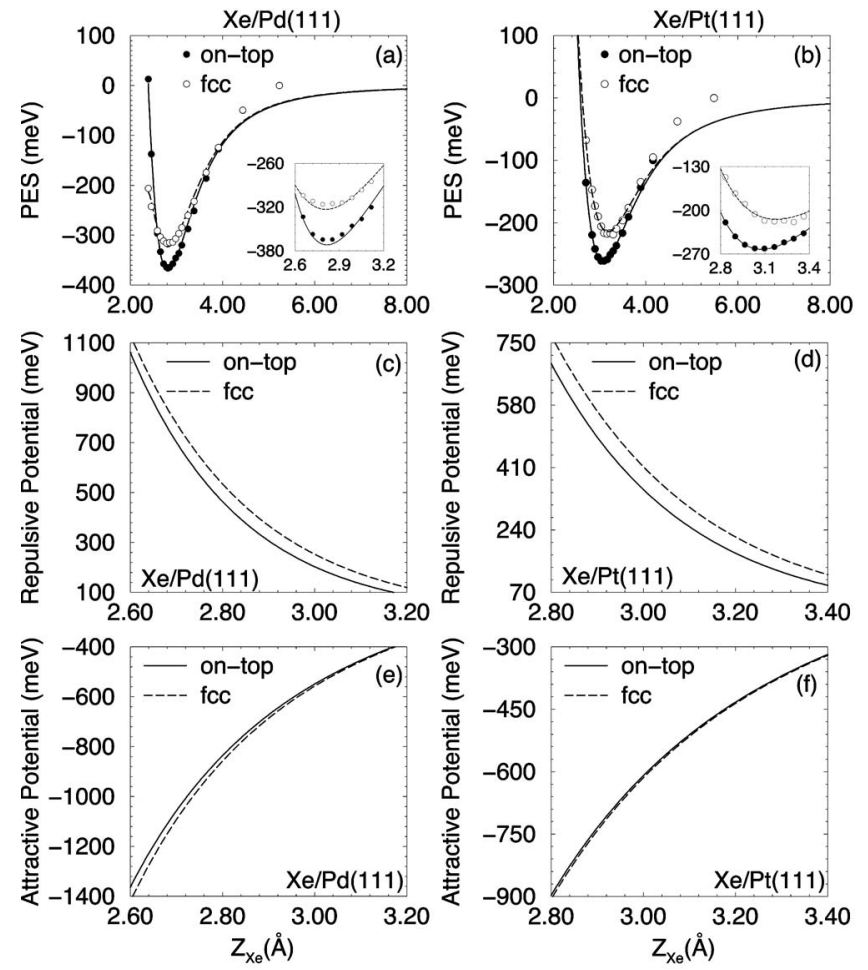

FIG. 4. The perpendicular potential energy surface (PES) for the (a) $\mathrm{Xe} / \mathrm{Pd}(111)$ and (b) $\mathrm{Xe} / \mathrm{Pt}(111)$ systems. The filled and open black circles indicate DFT-LDA values for Xe in the on-top and fcc sites in the $\sqrt{3}$ structure, respectively. The repulsive potential term of the PES for (c) $\mathrm{Xe} / \mathrm{Pd}(111)$ and (d) $\mathrm{Xe} / \mathrm{Pt}(111)$, and the attractive potential term of the PES for (e) $\mathrm{Xe} / \mathrm{Pd}(111)$ and (f) $\mathrm{Xe} / \mathrm{Pt}(111)$. In all cases, solid and dashed lines represent the fitted potential for Xe adsorbed in the on-top and fcc sites, respectively.

$0.03 \mathrm{meV}$. It is worthwhile pointing out that the PES minima do not correspond to the value of the adsorption energy since the latter is given with respect to the free Xe atom, and the PES with respect to a free Xe layer with $\sqrt{3}$ periodicity.

Further insight from the perpendicular PES can be obtained by a decomposition into attractive and repulsive contributions which can be achieved by fitting the DFT total energies to an analytical empirical function. One of the most successful functions, which is reported in Ref. 1, to describe the interaction between rare-gas atoms and surfaces is

$$
V_{\text {pes }}\left(Z_{\mathrm{Xe}}\right)=\alpha_{1} e^{-\alpha_{2} Z_{\mathrm{Xe}}}-\frac{C_{3}}{\left(Z_{\mathrm{Xe}}-Z_{0}\right)^{3}},
$$

where the first and second terms on the right-hand side of Eq. (2) describe the repulsive and attractive potential parts, respectively. The parameters, $\alpha_{1}, \alpha_{2}, C_{3}$, and $Z_{0}$ are determined by the fitting of the function to the DFT results. We recognize that the attractive potential in Eq. (2) is assumed to be due to van der Waals attraction, however, near the minimum in the PES there will be notable wave-function overlap, and as mentioned above, it has been shown that DFT can describe the physics in this region. Then from comparison of the fitted function and the DFT results, it can then be determined at which Xe adatom height above the surface the DFT 
TABLE VI. Perpendicular vibrational energy, $E_{\text {vib }}$, given in $\mathrm{meV}$, of Xe adatoms on metal surfaces in the $\sqrt{3}$ structure.

\begin{tabular}{|c|c|c|c|c|c|}
\hline \multirow[b]{2}{*}{$\mathrm{Xe} / \mathrm{metal}$} & \multicolumn{2}{|c|}{ LDA } & \multicolumn{2}{|c|}{ PBE } & \multirow[b]{2}{*}{ Expt. } \\
\hline & on-top & fcc & on-top & fcc & \\
\hline $\operatorname{Ti}(0001)$ & 3.99 & 3.41 & & & \\
\hline $\mathrm{Cu}(111)$ & 4.52 & 4.25 & 0.63 & 0.61 & $2.71^{\mathrm{a}}$ \\
\hline $\operatorname{Pd}(111)$ & 8.23 & 5.64 & 2.63 & 1.89 & \\
\hline $\operatorname{Pt}(111)$ & $5.73,5.6^{\mathrm{b}}, 8.5^{\mathrm{c}}$ & 4.76 & $1.85,2.0^{\mathrm{b}}$ & 1.76 & $3.80^{\mathrm{a}}$ \\
\hline
\end{tabular}

${ }^{\mathrm{a}}$ Reference 62 .

${ }^{\mathrm{b}}$ Reference 33 .

${ }^{\mathrm{c}}$ Reference 29.

PES does not follow the correct $1 /\left(Z_{\mathrm{Xe}}\right)^{3}$ behavior. The obtained empirical function and its decomposition into attractive and repulsive components are shown in Fig. 4. The coefficients $C_{3}$ and $Z_{0}$ are called the van der Waals constant and the van der Waals reference plane, respectively. Further details, and the values of the parameters and comparison with experiment and other work, can be found in Ref. 50.

It can seen that Eq. (2) fits the DFT results very closely around the equilibrium position, however for $\mathrm{Xe}$ adatom heights further from the surface, there is a significant deviation which is due to the fact that standard DFT within LDA or PBE cannot describe the asymptotic behavior of the PES of rare-gas atoms adsorbed on surfaces. Importantly, the repulsive potential term for $\mathrm{Xe}$ in the on-top site is weaker compared to Xe in the fcc site [see Figs. 4(c) and 4(d)]. Far from the surface the repulsive potential term is almost zero and equal for both sites. This result explains why the Xe adatoms can get closer to the surface on-top sites, i.e., since the repulsive potential term is weaker for Xe in the on-top sites. To investigate whether the site dependence of the repulsive potential term is an effect of the empirical PES function or a physical result, we use a different function. It is known that the attractive potential term should obey the van der Waals attraction for distances far from the surface, hence the main point in question concerns the exponential form for the repulsive term. We therefore use the Lennard-Jones repulsive term, $\alpha_{3} /\left(Z_{\mathrm{Xe}}\right)$ (Ref. 9) and find the same result, i.e., that the repulsive term is weaker for $\mathrm{Xe}$ in the on-top sites.

Intuitively it may be expected that the Pauli repulsion scales with the charge density, which is larger closer to the nuclei and hence larger for the on-top geometry. It has been shown, however, in relation to explaining the origin of the so-called anticorrugation effect, that this is a misconception and is not generally the case. ${ }^{32}$ The more appropriate picture is that the Pauli repulsion is strongest where the electron density is high and rigid. A high density, e.g., due to $d_{z^{2}}$-states would give a modest Pauli repulsion if the $d_{z^{2}}$ electrons could be easily transferred to $s$-like (or other) states, which is indeed the case here, as discussed later.

\section{Vibrational energy}

From the perpendicular PES described in the preceding section, the vertical vibrational energy of Xe adatoms on metal surfaces in the $\sqrt{3}$ structure can be calculated employing the harmonic and frozen substrate approximations. The perpendicular vibrational energy is calculated as

$$
E_{\mathrm{vib}}=\hbar \omega_{\perp}=\hbar \sqrt{\frac{f_{0}}{M_{\mathrm{Xe}}}},
$$

where $f_{0}$ is the force constant, $M_{\mathrm{Xe}}$ is the Xe mass, and $\hbar$ $=h / 2 \pi$, where $h$ is Planck's constant. The force constants are obtained from the fitted function in Eq. (2) using only the points around the equilibrium position. The perpendicular vibrational energies, $E_{\text {vib }}$, are summarized in Table VI.

The following trend can be noticed, $E_{\mathrm{vib}}^{\mathrm{Xe} / \mathrm{Pd}(111)}$ $>E_{\mathrm{vib}}^{\mathrm{Xe} / \mathrm{Pt}(111)}>E_{\mathrm{vib}}^{\mathrm{Xe} / \mathrm{Cu}(111)}>E_{\mathrm{vib}}^{\mathrm{Xe} / \mathrm{Ti}(0001)}$, i.e., largest for $\mathrm{Xe}$ on $\mathrm{Pd}(111)$ and smallest for $\mathrm{Xe}$ on $\mathrm{Ti}(0001)$. This corresponds to the trend obtained for the adsorption energies, and is in accord with the usual expectation that a stronger bond strength has a higher vibrational frequency. The vibrational energy is larger for $\mathrm{Xe}$ in the on-top sites, as obtained by both the LDA and PBE functionals, which is also consistent with the relative adsorption energies of $\mathrm{Xe}$ in on-top and fcc sites. The magnitude of the vibrational energy is, however consistently larger for the LDA than for the PBE functional (see Table VI). It can also be noticed that for $\mathrm{Xe}$ on $\mathrm{Cu}(111)$ and $\operatorname{Pt}(111)$, for which there are experimental results with which to compare, the LDA and PBE predict larger and smaller vibrational energies compared to the experimental results, respectively, however, the experimental trend, $E_{\mathrm{vib}}^{\mathrm{Xe} / \mathrm{Pt}(111)}$ $>E_{\mathrm{vib}}^{\mathrm{Xe} / \mathrm{Cu}(111)}$, is well described by our results.

Our calculated vibrational energies for $\mathrm{Xe} / \mathrm{Pt}(111)$ are in excellent agreement with the results reported by Betancourt and Bird, ${ }^{33}$ however, Müller ${ }^{29}$ reported a larger vibrational energy compared to our result (see Table VI), which might be to due to the cluster approach used to simulate the interaction between $\mathrm{Xe}$ and $\mathrm{Pt}(111)$, i.e., no lateral interactions between the Xe adatoms and the substrate is not laterally infinite. From the small values of the calculated vibrational energies, it can be concluded that they do not play a role in determining the Xe adsorption site preference, i.e., the difference in the relative adsorption for Xe in the on-top and fcc sites is considerably larger than the difference in the respective vibrational energies. 

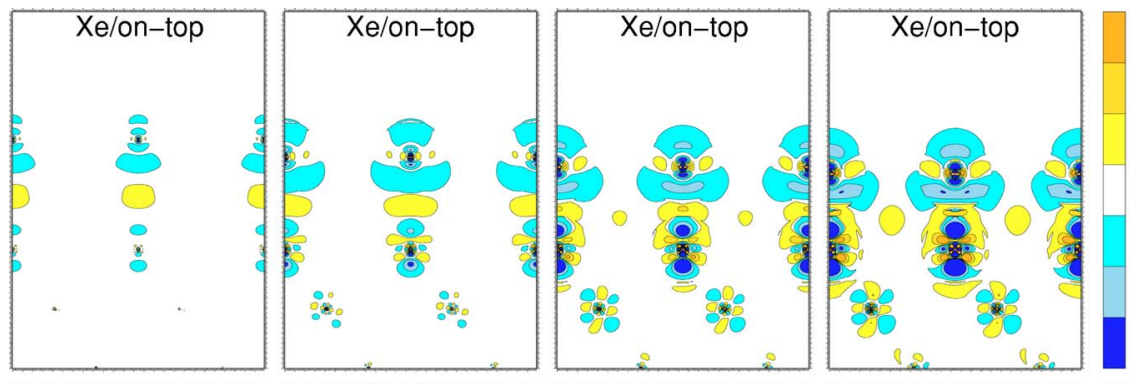

$+4.00$

$+2.00$

$+0.30$

$-0.30$

$-2.00$

$-4.00$
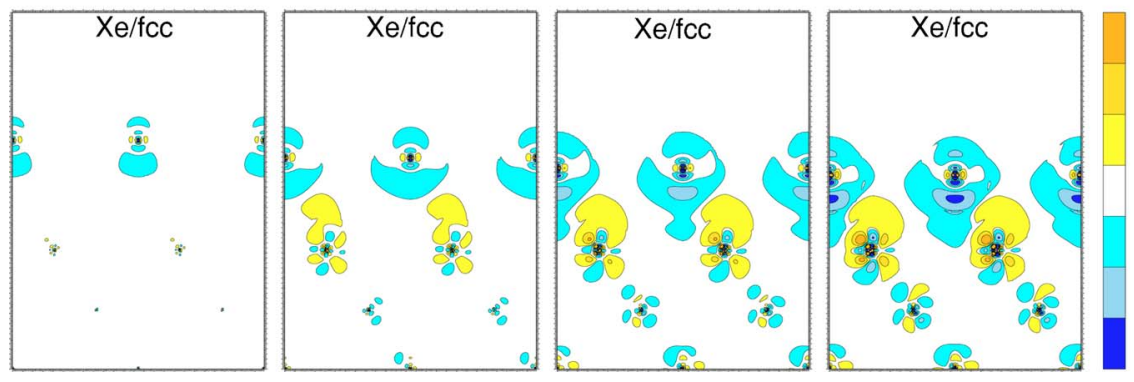

$+4.00$

$+2.00$

$+0.30$

$-0.30$

$-2.00$

$-4.00$
FIG. 5. (Color online) Difference electron density, $n^{\Delta}(\mathbf{r})=n^{\mathrm{Xe} / \mathrm{Pt}(111)}(\mathbf{r})$ $-n^{\mathrm{Pt}(111)}(\mathbf{r})-n^{\mathrm{Xe}-\text { layer }}(\mathbf{r})$, plots in units of $10^{-3} \mathrm{e} / \mathrm{bohr}^{3}$, in the $(11 \overline{2})$ plane, for $\mathrm{Xe}$ on $\mathrm{Pt}(111)$ in the $\sqrt{3}$ structure for two different sites, on-top (upper) and fcc (lower), employing the LDA. In the figures, from left to right, the Xe adatoms are placed at $Z_{\mathrm{Xe}}=4.15,3.49,3.10$, and $2.83 \AA$ above the surface, respectively. Yellow, gold, and orange (cyan, skyblue, and blue) colors indicate regions where the electron density increases (decreases).

\section{E. Difference electron density}

An important quantity which helps to characterize the orbitals that are involved in the interaction between adsorbate and substrate, is the difference electron density ${ }^{69}$ which is defined as

$$
n^{\Delta}(\mathbf{r})=n_{\mathrm{tot}}^{\mathrm{Xe} / \mathrm{metal}}(\mathbf{r})-n_{\mathrm{tot}}^{\mathrm{metal}}(\mathbf{r})-n_{\mathrm{tot}}^{\mathrm{Xe}-\mathrm{layer}}(\mathbf{r}),
$$

where $n_{\text {tot }}^{\mathrm{Xe} / \mathrm{metal}}(\mathbf{r})$ is the total electron density of the Xe/metal system, and $n_{\text {tot }}^{\text {metal }}(\mathbf{r})$ and $n_{\text {tot }}^{\text {Xe-layer }}(\mathbf{r})$ are the total electron density of the clean surface and the free Xe-layer, respectively.

\section{1. $n^{\Delta}(\mathrm{r})$ for different Xe heights above the surface}

We calculate $n^{\Delta}(\mathbf{r})$ for four different Xe heights above the surface, namely, $Z_{\mathrm{Xe}}=4.15,3.49,3.10$, and $2.89 \AA$, which are defined with respect to the topmost surface layer. The results are shown in Fig. 5 for Xe in the on-top and fcc sites. We restrict ourselves to the $\mathrm{Xe} / \mathrm{Pt}(111)$ system, since similar results are found for all studied systems.

The difference electron density plots have almost the same pattern of redistribution for the various adatom heights above the surface, and the main difference between them is in the magnitude of the induced electron density redistribution, which is weakest (strongest) for Xe adatoms at $4.15 \AA$ $(2.83 \AA)$ above the surface. A polarization of the Xe atom sets in when it is already far from the surface, where the negative end of the dipole points towards the surface, i.e., the electron density is enhanced closer to the metal surface (see Fig. 5, upper far left). This is due to the general property that the potential in front of a metal is attractive for electrons; specifically, the exchange-correlation potential is more attractive on the metal side of the $\mathrm{Xe}$ adatom than on the vacuum side.

At this large distance where the Xe dipole moment starts to build up, the electrostatic and covalent contributions are small, if present at all. This behavior is consistent with the pioneering works of Lang $^{30}$ and Antoniewicz. ${ }^{70}$ A corresponding polarization occurs in the surface, which can be described as the image of the Xe dipole. The build-up of these dipoles happens simultaneously (and continuously with decreasing distance from the surface) and are related to the partial population and depopulation of the Xe states (see Fig. 8 in which the local density of states are presented).

Figure 5 furthermore shows that the induced electron density redistributions on the $\mathrm{Xe}$ adatom and on the $\mathrm{Pt}$ atoms in the topmost layer is not the same for the on-top and fcc sites, and in fact the electron density redistribution on the metal atoms is clearly stronger for the on-top site. For the on-top site, there is a decrease of the electron density in the $\mathrm{Pt}$ $d$-states perpendicular to the surface, i.e. $\left(d_{z^{2}}\right.$-state $)$, while there is an increase in the electron density in the diagonal $d$-states $\left(d_{x z^{-}}\right.$and $d_{y z}$-states). This finding was also verified by decomposition of the electron density inside the muffintin sphere. On the Xe adatom there is a clear decrease in the electron density of the $\mathrm{Xe} p$-states, in particular of the $\mathrm{Xe}$ $p_{z}$-state (see also local density of states analyses below).

\section{2. $n^{\Delta}(\mathrm{r})$ for Xe at the equilibrium geometry}

For all studied systems there is a depletion of electron density about the Xe adatom which is weighted towards the surface. There is also an enhancement of electron density below this region between the Xe adatom and the topmost surface layer. These redistributions give rise to an induced surface dipole moment which points out of the surface, thus, it results in a decrease of the substrate work function, as discussed (see Table IV). As noted, the electron density redistributions are stronger for Xe in the on-top site, in accord with the observed greater work function change.

It can be noted that there is a small electron density accumulation close to the center of the Xe adatom (the small yellow region inside the larger depletion region) for all studied $\mathrm{Xe}$ and/or metal systems. It is largest for the $\mathrm{Xe} / \mathrm{Pd}(111)$ system with Xe in the on-top site. Similar behavior was observed by Wimmer et al. ${ }^{71}$ for $\mathrm{Cs} / \mathrm{W}(001)$. This electron density increase is due to the orthogonalization of $5 s$ - and $4 d$-states of the Xe adatom to the states of the substrate. 

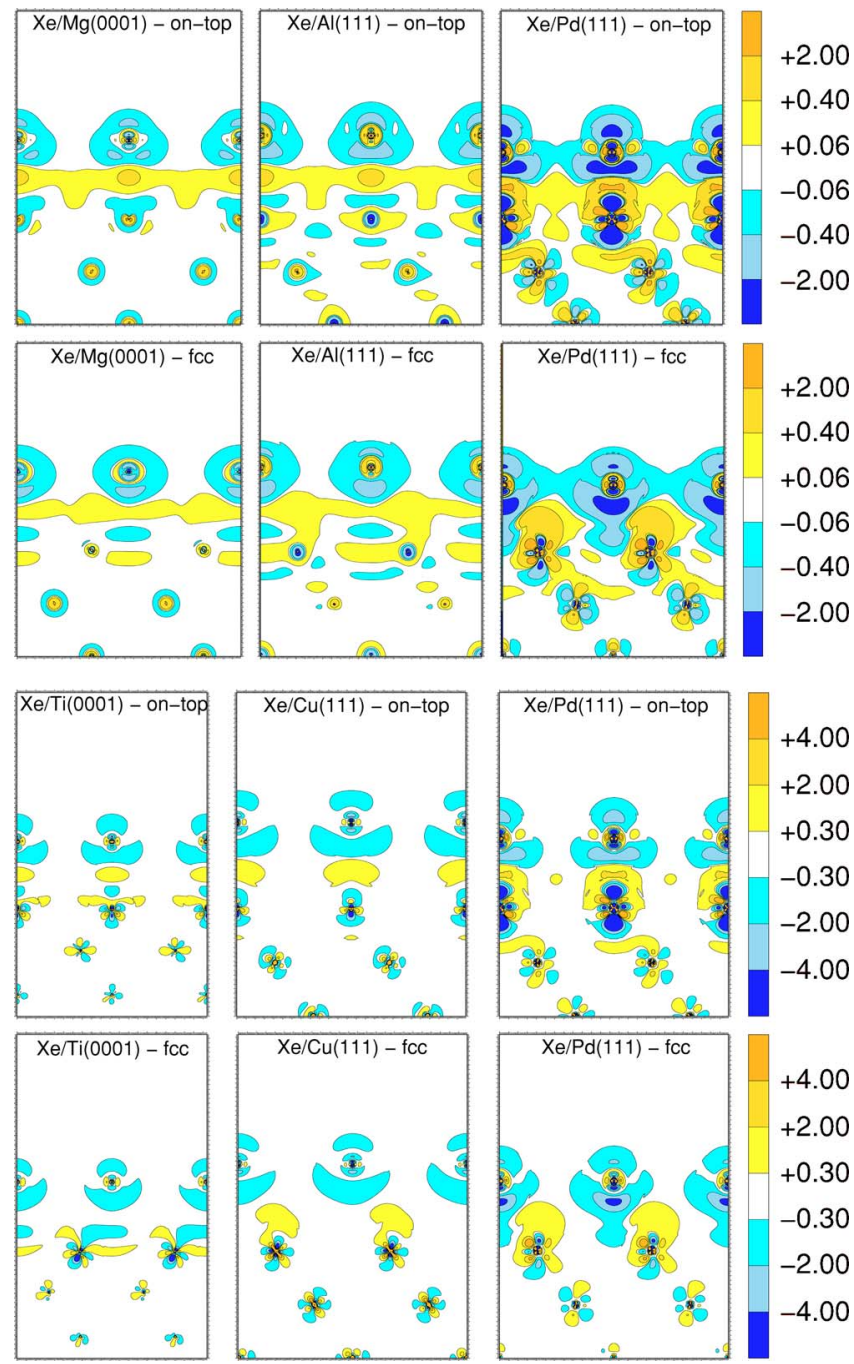

FIG. 6. (Color online) Difference electron density, $n^{\Delta}(\mathbf{r})$ $=n^{\mathrm{Xe} / \mathrm{metal}}(\mathbf{r})-n^{\text {metal }}(\mathbf{r})-n^{\mathrm{Xe}-\text { layer }}(\mathbf{r})$, plots in units of $10^{-3} e / \mathrm{bohr}^{3}$, for $\mathrm{Xe}$ on the $\mathrm{Mg}(0001), \mathrm{Al}(111), \mathrm{Ti}(0001), \mathrm{Cu}(111)$, and $\mathrm{Pd}(111)$ surfaces in the $\sqrt{3}$ structure in the $(2 \overline{1} 10)$ plane for the $h c p(0001)$ surfaces and in the $(11 \overline{2})$ plane for the fcc(111) surfaces, calculated by the LDA at the equilibrium geometry for the on-top and fcc sites. Yellow, gold, and orange (cyan, skyblue, and blue) indicate regions of electron density increase (decrease). Note that the $\mathrm{Xe} / \mathrm{Pd}(111)$ system is shown twice (with different scales) for reference.

From comparison of the difference electron density plots for $\mathrm{Xe}$ on $\mathrm{Mg}(0001), \operatorname{Al}(111)$, and $\mathrm{Pd}(111)$ shown in Fig. 6, we can see that the magnitude of the redistributions are smaller for $\mathrm{Xe}$ on $\mathrm{Mg}(0001)$ and $\mathrm{Al}(111)$ as compared to $\operatorname{Pd}(111)$, which is understandable since the interaction is weaker. Furthermore, we note that the electron density redistribution on the topmost surface metal layer for free-electronlike metal surfaces, exhibits different features to the transition metals due to the lack of occupied $d$-states. As will be seen below, the work function change of the $\operatorname{Mg}(0001)$ and $\mathrm{Al}(111)$ surfaces is very small, which is in accord with the observed small electron density redistributions, the weak adsorption energy, and the large Xe-metal distance. For $\mathrm{Xe} / \mathrm{Mg}(0001)$ the main changes in the electron density of the metal atoms occur in the delocalized $s$-states. For
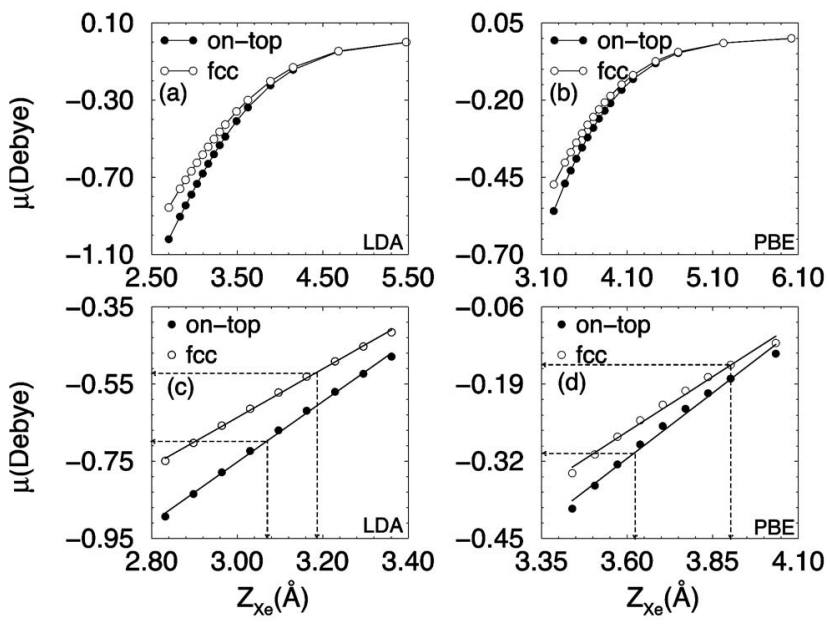

FIG. 7. Induced dipole moment, $\mu$, for the $\mathrm{Xe} / \mathrm{Pt}(111)$ system in the $\sqrt{3}$ structure as function of the Xe height above the surface, $Z_{\mathrm{Xe}}$, for the on-top and fcc sites obtained by the LDA (left) and PBE (right) functionals. (a) and (b) show $\mu$ for a large range of $Z_{\mathrm{Xe}}$ values, where the continuous lines are a guide for the eyes. (c) and (d) show $\mu$ only for $Z_{\mathrm{Xe}}$ close to the equilibrium position to show the linear behavior.

$\mathrm{Xe} / \mathrm{Al}(111)$, the electron density redistribution is similar to $\mathrm{Xe} / \mathrm{Mg}(0001)$, except that on the metal atoms there is a slightly larger increase in the electron density, which is due to population of the $p$-states of the $\mathrm{Al}$ atoms. Although the discussion above is based entirely on the LDA results, the same pattern for the induced electron density difference distributions is found for the PBE functional, which are reported in Ref. 50. The magnitude of the PBE induced electron density is, however, smaller than the LDA results which is consistent with the larger equilibrium Xe vertical distance and weaker adsorption energies and smaller changes in the work function. The electron density redistributions for the $\mathrm{Xe} / \mathrm{Cu}$ and $\mathrm{Xe} / \mathrm{Ti}$ systems are qualitatively similar to $\mathrm{Xe} / \mathrm{Pt}$ and $\mathrm{Xe} / \mathrm{Pd}$, except the magnitudes are smaller due to the weaker interaction resulting from the full $d$-shell $(\mathrm{Cu})$ and the small occupancy of the (Ti) $d$-states.

\section{F. Induced dipole moment}

We calculate the induced dipole moment, $\mu$, for Xe adatoms on metal surfaces in the $\sqrt{3}$ structure using the Helmholtz equation, ${ }^{72-74}$

$$
\mu\left(Z_{\mathrm{Xe}}, \Theta_{\mathrm{Xe}}\right)=\frac{1}{12 \pi} \frac{A_{(1 \times 1)}}{\Theta_{\mathrm{Xe}}} \Delta \Phi\left(Z_{\mathrm{Xe}}, \Theta_{\mathrm{Xe}}\right),
$$

which relates the induced work function change, $\Delta \Phi\left(Z_{\mathrm{Xe}}, \Theta_{\mathrm{Xe}}\right)=\Phi^{\mathrm{Xe} / \mathrm{metal}}\left(Z_{\mathrm{Xe}}, \Theta_{\mathrm{Xe}}\right)-\Phi^{\text {metal }}$, to an array of adsorbates where each adsorbate is represented by a point dipole moment normal to the surface. In Eq. (5), $A_{(1 \times 1)}$ is the area of the $(1 \times 1)$ surface unit cell in $\AA^{2}$. If $\Delta \Phi$ is given in eV, Eq. (5) yields $\mu$ in Debye. $\Theta_{\mathrm{Xe}}=1 / 3$ for the $\sqrt{3}$ structure, hence, only the induced dipole moment versus $Z_{\mathrm{Xe}}$ will be shown in the figures. 
TABLE VII. Induced dipole moment, $\mu$, for Xe adatoms in the on-top and fcc sites on metal surfaces in the $\sqrt{3}$ structure at the equilibrium geometries. $\mu$ is given in Debye.

\begin{tabular}{|c|c|c|c|c|c|}
\hline \multirow[b]{2}{*}{$\mathrm{Xe} / \mathrm{metal}$} & \multicolumn{2}{|c|}{ LDA } & \multicolumn{2}{|c|}{ PBE } & \multirow[b]{2}{*}{ Expt. } \\
\hline & on-top & fcc & on-top & fcc & \\
\hline $\operatorname{Mg}(0001)$ & -0.15 & -0.11 & & & \\
\hline $\mathrm{Al}(111)$ & -0.21 & -0.18 & & & $-0.24^{\mathrm{a}}$ \\
\hline $\operatorname{Ti}(0001)$ & -0.49 & -0.40 & -0.20 & -0.17 & \\
\hline $\mathrm{Cu}(111)$ & -0.40 & -0.35 & -0.11 & -0.09 & $-0.24^{\mathrm{a}}$ \\
\hline $\operatorname{Pd}(111)$ & -0.74 & -0.65 & -0.44 & -0.33 & $-0.70^{\mathrm{a}}$ \\
\hline $\operatorname{Pt}(111)$ & -0.71 & -0.51 & -0.31 & -0.16 & $-0.30^{\mathrm{a}}$ \\
\hline
\end{tabular}

${ }^{a}$ Reference 62.

\section{Dipole moment for different Xe adlayer heights above the surface}

Here we will restrict our discussion to the $\mathrm{Xe} / \mathrm{Pt}(111)$ system, since similar behavior is found for the other systems. The induced dipole moment for Xe in the on-top and fcc sites as a function of the Xe height above the metal surface is shown in Fig. 7. The relationships between $\mu$ and $\Delta \Phi$ are $\mu=0.521 \Delta \Phi$ (LDA) and $\mu=0.543 \Delta \Phi$ (PBE). We find that $\mu$ decreases linearly with increasing separation between Xe and $\operatorname{Pt}(111)$ for $Z_{\mathrm{Xe}}$ values close to the equilibrium geometry [see Figs. 7(c) and 7(d)]. The continuous lines in Figs. 7(c) and 7 (d) were obtained by fitting straight lines to these points. ${ }^{50}$

The induced dipole moment is greater for Xe in the on-top site for all distances. Thus, the larger value of $\mu$ (and substrate work function change) at the equilibrium geometry for the on-top site is not simply because the Xe adatom gets closer to the surface. Furthermore, the large deviations between the calculated work function changes and the experimental ones can be understood; namely, because a relatively small error in the equilibrium Xe vertical distance induces a large error in the induced dipole moment and hence in the work function change.
From Figs. 7(a) and 7(b), it can be seen that the dipole moment starts to build up far from the surface and it increases smoothly in magnitude as the Xe adatom approaches the surface. For example, for Xe in the on-top site at $4.15 \AA$ (i.e., $1.08 \AA$ above the equilibrium position), the substrate work function decreases by $0.25 \mathrm{eV}$, which corresponds to an induced dipole moment of -0.13 Debye. Thus, the mechanism that gives rise to the induced dipole moment starts to act relatively far from the surface. This can also be seen from Fig. 5, showing the difference electron density distributions as a function of Xe height above the surface, where there is already significant redistributions taking place at this distance from the surface.

\section{Dipole moment for Xe at the equilibrium geometry on different metal surfaces}

The induced dipole moments, $\mu$, at the equilibrium adsorbate-substrate geometry are summarized in Table VII. The largest induced dipole moment occurs for $\mathrm{Xe} / \mathrm{Pd}(111)$, while the smallest is for $\mathrm{Xe} / \mathrm{Mg}(0001)$. In all cases the induced dipole moment is larger for Xe in the on-top site. The

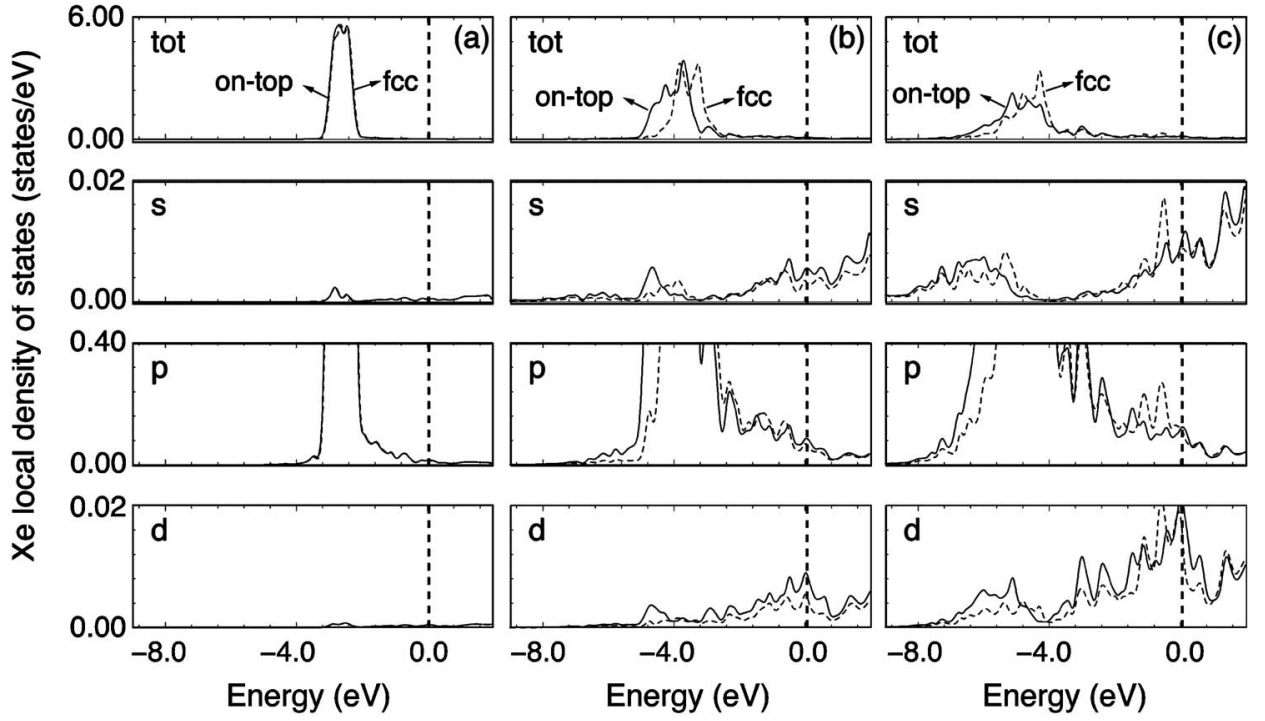

FIG. 8. Local density of states and decomposition into states with $s, p$, and $d$ character for the $\mathrm{Xe} / \mathrm{Pt}(111)$ system in the $\sqrt{3}$ structure calculated with the LDA for three $\mathrm{Xe}$ adatom heights, (a) $Z_{\mathrm{Xe}}$ $=4.53 \AA$; (b) $Z_{\mathrm{Xe}}=3.07 \AA$ (equilibrium position); (c) $Z_{\mathrm{Xe}}=2.70 \AA$. The solid (dashed) lines indicate $\mathrm{Xe}$ adatoms in the on-top (fcc) sites, while the vertical dashed lines indicate the Fermi level. 
trend is the same for the LDA or PBE functionals, only the magnitude is larger for the LDA functional.

\section{G. Local density of states}

To gain more insight into the change in the Xe electronic states due to adsorption on the surface, and the nature of the Xe-metal interaction in general, we calculate the local density of states (LDOS).

\section{LDOS for different Xe adatom heights above the surface}

Here we will again restrict ourselves to $\mathrm{Xe} / \mathrm{Pt}(111)$ since the behavior is the same for the other systems. We consider three Xe adatom heights above the surface, 4.53, 3.07 (equilibrium distance), and $2.70 \AA$. The Xe LDOS of Xe adatoms in the on-top and fcc sites are plotted in Fig. 8. A first inspection of Fig. 8 shows that the center of gravity of the Xe LDOS are shifted several $\mathrm{eV}$ below the Fermi level, which is understandable because the effective potential at the surface is lower than that in the vacuum region. Furthermore, there is a clear broadening of the states as the Xe adatom approaches the surface (from left to right in Fig. 8) which will be discussed below.

The Xe LDOS in the range plotted in Fig. 8 is mainly due to Xe states with $p$ character. The electronic configuration of the free $\mathrm{Xe}$ atom is $[\mathrm{Kr}] 4 d^{10} 5 s^{2} 5 p^{6}$. The $\mathrm{Xe} 4 d$ - and $5 s$-states are 54.9 and $15.5 \mathrm{eV}$ below the Fermi level, respectively, for $\mathrm{Xe}$ in the on-top sites at the equilibrium geometry and so are outside the range displayed in Fig. 8. The states with $s$ and $d$ character shown in Fig. 8 are therefore contributions from the previously unoccupied $\mathrm{Xe}$ states, i.e., $6 s$ - and $5 d$-states, which broaden upon Xe adsorption on the surface where the tails extend several $\mathrm{eV}$ below the Fermi level. Furthermore, it can be seen that there is a depopulation of the Xe $5 p$-states, since the tail of these states with $p$ character extends above the Fermi level. By decomposition of the Xe LDOS with $p$ character into $p_{z}, p_{x}$, and $p_{\mathrm{y}}$ components, it is found that the largest depopulation occurs for the $p_{z}$-states. These effects are observed for both the on-top and fcc sites, where the magnitude of the depopulation is greater for the on-top site. These changes in the LDOS are consistent with the difference electron densities shown in Fig. 5.

The existence of a partial occupation of the Xe $6 s$-state was the basis of the " $s$-resonance model" proposed by Wandelt and Gumhalter ${ }^{75}$ and used to discuss the face specificity of the $\mathrm{Xe} / \mathrm{Pd}$ bond. Furthermore, it was attributed to being the origin of the Fermi level Xe LDOS by Eigler et al. ${ }^{76}$ which renders Xe visible on $\mathrm{Ni}(110)$ in STM experiments. The mentioned works are consistent with our present results, however, we want to add that at the Fermi level there are also Xe states with $s, p$, and $d$ character, and not only with $s$ character. The occupation of the Xe $6 s$ - and $5 d$-states, and the depopulation of the Xe $5 p$-state can be explained as an effect of the Pauli exclusion principle. Overlap of the Xe and substrate wave functions can only occur with depopulation of the highest occupied Xe states and population of the lowest unoccupied Xe states, since the Xe atom has a closed shell. Therefore, in the interaction mechanism between Xe and the metal surfaces, there is a transition like $5 p$
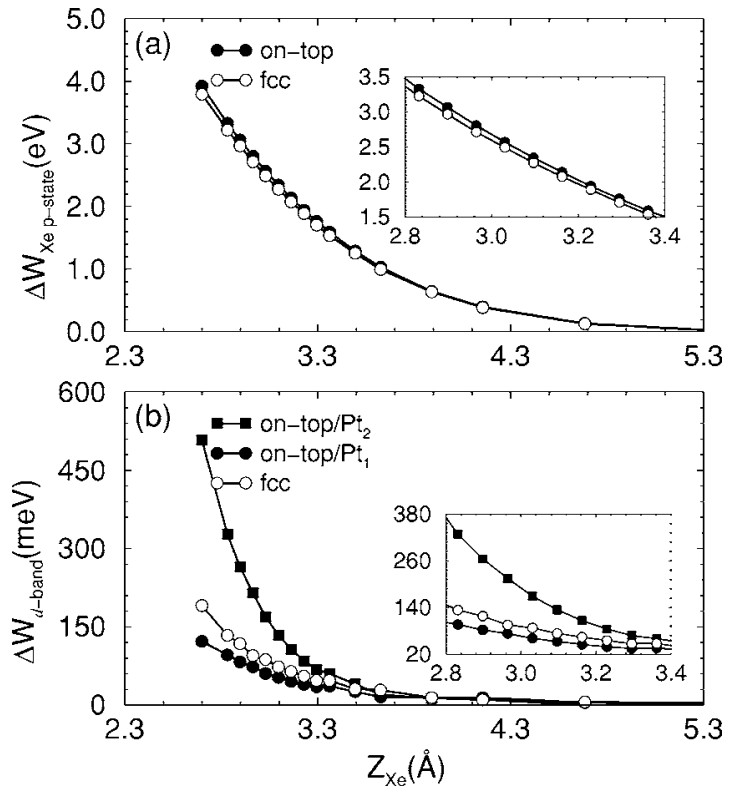

FIG. 9. (a) Bandwidth of the Xe $5 p$-state for Xe on the Pt(111) surface in the on-top and fcc sites calculated with respect to the bandwidth of the isolated Xe layer as a function of Xe distance from the surface, i.e., $\Delta W_{\mathrm{Xe}-5 p}=W_{\mathrm{Xe}-5 p}^{\mathrm{Xe} / \mathrm{metal}}-W_{\mathrm{Xe}-5 p}^{\mathrm{Xe}-\mathrm{Xayer}}$, where $W_{\text {Xe--5per }}^{\text {Xe-lay }}=0.61 \mathrm{eV}$. (b) Bandwidth of the $d$-state of $\mathrm{Pt}$ atoms in the topmost layer with respect to the bandwidth of the $d$-states of the clean surface, i.e., $\Delta W_{d \text {-band }}=W_{d \text {-band }}^{\mathrm{Xe} / \mathrm{Pt}(11)}-W_{d \text {-band }}^{\mathrm{Pt}(111)}$ (where $W_{d \text {-band }}^{\mathrm{Pt}(111)}$ $=6.13 \mathrm{eV}$ ), as a function of Xe distance above the surface. For Xe in the on-top sites, $\mathrm{Pt}_{1}$ and $\mathrm{Pt}_{2}$ indicate $\mathrm{Pt}$ atoms not under and under the Xe adatoms, respectively. For Xe in the fcc site, the three $\mathrm{Pt}$ atoms are equivalent.

$\rightarrow(6 s 5 d)$, where the dominant contribution is $5 p_{z}$ $\rightarrow\left(6 s 5 d_{z^{2}}\right)$.

In Fig. 8 we show the bandwidth of the Xe $5 p$-states and the Pt $d$-states for different Xe adatom heights. It can be seen clearly that the bandwidth increases as Xe approaches the metal surface. The inset in Fig. 9(a) shows that the largest changes occur for $\mathrm{Xe}$ in the on-top site. For example, at $Z_{\mathrm{Xe}}=4.53,3.07$, and $2.70 \AA$, the bandwidths of the $\mathrm{Xe}$ $5 p$-state are $1.05 \mathrm{eV}$ (on-top), $1.04 \mathrm{eV}$ (fcc), $3.00 \mathrm{eV}$ (ontop), $2.92 \mathrm{eV}$ (fcc), $4.58 \mathrm{eV}$ (on-top), and $4.44 \mathrm{eV}$ (fcc), respectively. The bandwidth of the Xe $5 p$-state of a free Xe layer is $0.61 \mathrm{eV}$ which is considerably smaller than for even the largest distance $(4.53 \AA)$ considered, which shows that there is already a considerable influence from the substrate.

The changes in the bandwidth of the $d$-states are smaller than for the Xe $5 p$-state [see Fig. 9(b)]. For Xe in the on-top sites, $\Delta W_{d \text {-band }}$ changes by a large amount for the Pt atom under the $\mathrm{Xe}$ adatom (labeled $\mathrm{Pt}_{2}$ ). For the $\mathrm{Pt}$ atoms not under the $\mathrm{Xe}$ adatom (labeled $\mathrm{Pt}_{1}$ ), the changes are smaller but the same trend is observed. For Xe in the hollow site, the changes in the bandwidth of the surface Pt atoms lie between those mentioned for the top site. The same trends are found for all the other systems.

\section{LDOS for Xe at the equilibrium geometry on different metal surfaces}

The relative bandwidths, $\Delta W_{\mathrm{Xe}-5 p}^{\mathrm{Xe} / \mathrm{metal}}$, and the center of gravities of the Xe $5 p$-state are listed in Table VIII. We found 
TABLE VIII. The bandwidth of the Xe $5 p$-state for Xe on the metal surfaces with respect to the bandwidth of the Xe $5 p$-state in the free layer, i.e., $\Delta W_{\mathrm{Xe}-5 p}=W_{\mathrm{Xe}-5 p}^{\mathrm{Xe}-{ }_{0}}-W_{\mathrm{Xe}-5 p}^{\mathrm{Xe}}$ free adlayer , for Xe in the on-top and fcc sites. The center of gravity of the Xe $5 p$-state with respect to the Fermi, $C_{\mathrm{Xe}-5 p}^{\epsilon_{F}}$, and vacuum, $C_{\mathrm{Xe}-5 p}^{\mathrm{vac}}$, levels for the on-top and fcc hollow sites. $\Delta W_{\mathrm{Xe}-5 p}, C_{\mathrm{Xe}-5 p}^{\epsilon_{F}}$, and $C_{\mathrm{Xe}-5 p}^{\mathrm{vac}}$ are given in electron volt (eV).

\begin{tabular}{|c|c|c|c|c|c|c|c|}
\hline \multirow[b]{2}{*}{$\mathrm{Xe} / \mathrm{metal}$} & & \multicolumn{2}{|c|}{$\Delta W_{\mathrm{Xe}-5 p}$} & \multicolumn{2}{|c|}{$C_{\mathrm{Xe}-5 p}^{\epsilon_{F}}$} & \multicolumn{2}{|c|}{$C_{\mathrm{Xe}-5 p}^{\mathrm{vac}}$} \\
\hline & & on-top & $\mathrm{fcc}$ & on-top & fcc & on-top & fcc \\
\hline $\operatorname{Mg}(0001)$ & LDA & 1.23 & 1.18 & 4.83 & 4.77 & 8.44 & 8.44 \\
\hline $\mathrm{Al}(111)$ & LDA & 1.22 & 1.16 & 4.63 & 4.58 & 8.46 & 8.46 \\
\hline \multirow[t]{2}{*}{$\operatorname{Ti}(0001)$} & LDA & 1.66 & 1.42 & 4.66 & 4.54 & 8.49 & 8.48 \\
\hline & PBE & 0.86 & 0.77 & 4.21 & 4.14 & 8.25 & 8.25 \\
\hline \multirow[t]{2}{*}{$\mathrm{Cu}(111)$} & LDA & 1.27 & 1.19 & 4.15 & 4.06 & 8.48 & 8.47 \\
\hline & PBE & 0.34 & 0.26 & 3.68 & 3.63 & 8.23 & 8.22 \\
\hline \multirow[t]{2}{*}{$\operatorname{Pd}(111)$} & LDA & 2.73 & 2.67 & 4.38 & 4.21 & 8.55 & 8.55 \\
\hline & PBE & 1.59 & 1.27 & 3.87 & 3.65 & 8.26 & 8.25 \\
\hline \multirow[t]{2}{*}{$\operatorname{Pt}(111)$} & LDA & 2.39 & 2.31 & 3.80 & 3.39 & 8.50 & 8.47 \\
\hline & PBE & 1.12 & 0.68 & 3.11 & 2.81 & 8.24 & 8.23 \\
\hline
\end{tabular}

that $\Delta W_{\mathrm{Xe}-5 p}^{\mathrm{Xe} / \mathrm{metal}}$ increases from the $\mathrm{Xe} / \mathrm{Mg}(0001)$ system to $\mathrm{Xe} / \mathrm{Pd}(111)$. This makes sense since the interaction between $\mathrm{Xe}$ atoms and free-electron-like metal surfaces is very weak compared to the Xe-transition-metal interaction. It can be noted that these results largely correlate with the adsorption energy and the work function change, and (inversely) with that of the equilibrium Xe-metal distance.

The center of gravity of the Xe $5 p$-states are calulated with respect to the Fermi, $C_{\mathrm{Xe}-5 p}^{\epsilon_{F}}$, and vacuum, $C_{\mathrm{Xe}-5 p}^{\mathrm{vac}}$, levels. We found that $C_{\mathrm{Xe}-5 p}^{\epsilon_{F}}$ is different for each adsorption site and substrate. In particular, $C_{\mathrm{Xe}-5 p}^{\epsilon_{F}}$ is larger for Xe in the on-top sites for all studied systems (see Table VIII). However, we found that $C_{\mathrm{Xe}-5 p}^{\mathrm{vac}}$ is almost constant for all adsorption sites and substrates. To understand why $C_{\mathrm{Xe}-5 p}^{\mathrm{vac}}$ is constant for the different systems, we recall that $C_{\mathrm{Xe}-5 p}^{\mathrm{vac}}=C_{\mathrm{Xe}-5 p}^{\epsilon_{F}}+\Phi^{\mathrm{Xe} / \mathrm{metal}}$. And it was found that $\Phi^{\mathrm{Xe} / \mathrm{metal}}$ is larger for Xe in the fcc site, while $C_{\mathrm{Xe}-5 p}^{\epsilon_{F}}$ is larger for the on-top sites, hence, it gives rise to a constant value for $C_{\mathrm{Xe}-5 p}^{\mathrm{vac}}$. This behavior is in good agreement with the observation by Wandelt ${ }^{5}$ that the binding energy of the $\mathrm{Xe} 5 p_{1 / 2}$-state measured by photoemission, with respect to the vacuum level, is constant for a large number of metal substrates, namely, $\operatorname{Pd}(110), \operatorname{Pd}(100)$, Pd(111), Pt(111), Ru(0001), W(100), W(110), Ni(110), $\mathrm{Ni}(100), \mathrm{Ni}(111), \mathrm{Cu}(110), \mathrm{Al}(111)$, and $\mathrm{Ag}(111)$.

\section{H. Electronic reactivity function}

Following the concepts introduced by Fukui, ${ }^{77,78}$ Wilke et al. ${ }^{79}$ proposed a scheme to characterize the reactivity of metal surfaces, based on the study of changes in the electronic states close to the Fermi level due to the broadening of the occupation numbers in the Fermi distribution. The Fukui concepts are based on the analysis of the electronic structure of the reactants before they interact. Typically, it is useful in the study of reactions where the interactions between the reactants are weak, which is the case for Xe adatoms on metal surfaces. The "Wilke function" is defined as

$$
W(\mathbf{r})=\frac{1}{k_{B}^{2} T_{\mathrm{el}}}\left(\frac{\partial n\left(\mathbf{r}, T_{\mathrm{el}}\right)}{\partial T_{\mathrm{el}}}\right)_{V_{\mathrm{ext}}(\mathbf{r}), N} \approx \frac{n\left(\mathbf{r}, T_{\mathrm{el}, 2}\right)-n\left(\mathbf{r}, T_{\mathrm{el}, 1}\right)}{k_{B}^{2} T_{\mathrm{el}, 2}\left[T_{\mathrm{el}, 2}-T_{\mathrm{el}, 1}\right]}
$$

where the subscripts $V_{\text {ext }}$ and $N$ denote that the external potential and the number of electrons are conserved. $T_{\mathrm{el}}$ is the artificial electronic temperature and $k_{B}$ is the Boltzmann constant. In the "Wilke function" plots, the positive regions reveal the electron states that can be easily occupied, while negative regions show occupied states which can be most easily depopulated. Thus, it gives a spatially resolved picture of the information contained in the local density of states close to the Fermi level.

The $W(\mathbf{r})$ function is calculated according to the following procedure: (i) two self-consistent calculations using different broadening parameters are performed $\left(k_{B} T_{\mathrm{el}, 2}\right.$ $=123.8 \mathrm{meV}$ and $\left.k_{B} T_{\mathrm{el}, 1}=1.4 \mathrm{meV}\right)$ for a good mesh of k-points, e.g., $(12 \times 12 \times 1)$; (ii) the $\mathbf{k}$-point mesh is increased in both calculations to a highly dense k-point mesh $[(30 \times 30 \times 4)$, or $348 \mathbf{k}$-points in the IBZ]. By inspecting the "Wilke function" plots as a function of $\mathbf{k}$-points, we found
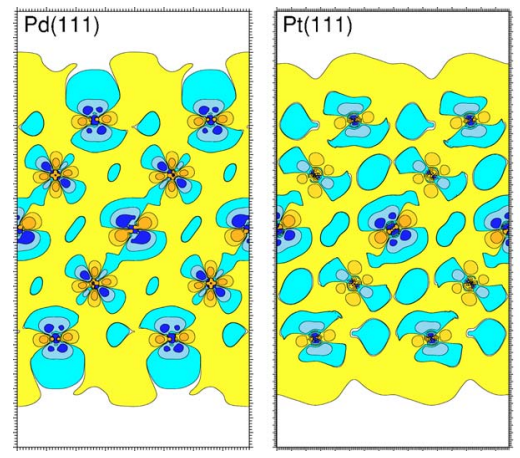

$+20.0$

$+5.0$

$+0.1$

$-0.1$

$-5.0$

$-20.0$

FIG. 10. (Color online) "Wilke function" plots, $W(\mathbf{r})$, in the $(11 \overline{2})$ plane perpendicular to the $\mathrm{Pd}(111)$ and $\mathrm{Pt}(111)$ surfaces [cf. Eq. (6)]. The units are in $\AA^{-3} \mathrm{eV}^{-2}$. 
that $W(\mathbf{r})$ function is very sensitive to the number of $\mathbf{k}$-points (see Ref. 50), so it is necessary to use such a dense mesh.

The $W(\mathbf{r})$ function plots are shown in Fig. 10, for the $(11 \overline{2})$ plane perpendicular to the $\operatorname{Pd}(111)$ and $\operatorname{Pt}(111)$ surfaces. We observe the following features: the metal $d$-states

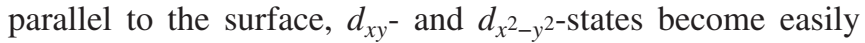
populated (yellow, gold, and orange regions), however the metal $d$-states perpendicular to the surface, $d_{z^{2}}$-state, and diagonal to the surface, $d_{x z^{-}}$and $d_{y z^{-}}$-states, become easily depopulated (cyan, skyblue, and blue regions). The magnitude of the population and depopulation of the $d$-state components is smaller for $\mathrm{Cu}(111)$, which is expected since the $\mathrm{Cu}$ $d$-band is completely occupied. ${ }^{50}$ The largest changes occur for $\operatorname{Pd}(111)$, which indicates that $\mathrm{Pd}(111)$ is the most reactive of the systems we studied, which is consistent with it having the largest adsorption energy for Xe.

It can also be seen in Fig. 10 that for Pd(111), from 2.0 to $3.0 \AA$ above the topmost surface layer, in the region just above the metal atoms, the electron density decreases, while it increases in the hollow site region. Thus, we find that the electronic character at the on-top region is "donorlike" and at the hollow region it is "acceptorlike." This trend is similar, but not exactly the same for $\operatorname{Pt}(111)$, for which there appears to be a larger contribution from the delocalized $s$-states, which spill out into the vacuum region.

For $\operatorname{Mg}(0001)$ and $\mathrm{Al}(111)$, there appears to be essentially no distinguishing differences in the plots of the $W(\mathbf{r})$ function at the on-top or hollow sites, ${ }^{50}$ and so it is hard to explain the site preference on the basis of reactivity arguments of the clean surface alone. Indeed for these surfaces, the on-top site is only favorable by a very small amount, which is at the limit of the accuracy of our calculations [i.e., by 4.43 and $1.12 \mathrm{meV}$, respectively, for $\mathrm{Xe} / \mathrm{Mg}(0001)$ and $\mathrm{Xe} / \mathrm{Al}(111)]$. For $\mathrm{Ti}(0001)$, an early transition metal in which only bonding $d$-states are occupied, the plot is qualitatively different to that of the described late transition metal surfaces. In particular, in contrast the $d_{z^{2}}$-states are not most easily depleted, but the more delocalized $s$ and $p$ electrons and the $d_{x y}$-states are. For this metal, the $d_{z^{2}}$-orbitals have a lower DOS at the Fermi level and are comparatively less reactive. This results in a "Wilke function" plot with a region of electron depletion spreading right over the fcc site, as well as over the on-top site, lying well above the $d_{z^{2}}$-states.

Thus, for $\mathrm{Ti}(0001)$ the reactivity analysis does not lead to a clear indication for the preference of the on-top site. The general electron density difference distribution for $\mathrm{Xe}$ in the on-top site on $\operatorname{Ti}(0001)$, is nevertheless, very similar to that of the other systems studied as can be seen from Fig. 5. In this case there is an even smaller perturbation of the metal states than for $\mathrm{Cu}(111)$, indicating a weaker interaction as is consistent with the smaller adsorption energy and the larger Xe-metal distance.

\section{CHEMICAL CONTRIBUTION TO THE BONDING}

From our analyses we do observe behavior characteristic of a chemical interaction between Xe and the metal surface, but this behavior could also be interpreted and explained in terms of polarization and Pauli repulsion effects. In particular, the bandwidth of the $s$, and $p$ band (transition metal and free-electron-like metal surfaces), and the $d$-band (transition metal surfaces) of the metal atoms in the topmost layer broaden as Xe approaches the metal surface, as do the Xe states, which was shown in Fig. 9. Such changes could be taken as indication of a hybridization of adsorbate and metal states such as occurs in covalentlike bonds, and indeed the changes in the bandwidth are larger for the transition metal surfaces, in particular, for $\operatorname{Pd}(111)$ and $\operatorname{Pt}(111)$, while they are very small for $\mathrm{Cu}(111)$, and even smaller for freeelectron-like metal surfaces, in correlation with the trend found for the adsorption energies.

Furthermore, if we compare the equilibrium Xe-metal distances for the on-top site which are 3.80, 3.69, 3.56, 3.26, 3.07, and $2.85 \AA$, for $\operatorname{Mg}(0001), \operatorname{Al}(111), \operatorname{Ti}(0001), \mathrm{Cu}(111)$, $\operatorname{Pt}(111)$, and $\operatorname{Pd}(111)$, respectively, with sum of the Xe and metal atom radii in bulk material $\left(R_{\mathrm{Xe}}^{\text {bulk }}+R_{\text {metal }}^{\text {bulk }}\right)$, which are 3.64, 3.48, 3.51, 3.31, 3.45, and $3.43 \AA$, for $\mathrm{Mg}, \mathrm{Al}, \mathrm{Ti}, \mathrm{Cu}$, $\mathrm{Pt}$, and $\mathrm{Pd}$, respectively. Thus, we can notice that for $\mathrm{Xe}$ on $\mathrm{Mg}(0001), \mathrm{Al}(111)$, and $\mathrm{Ti}(0001)$ the Xe-metal distance is larger than that obtained by the sum of the radii, while for Xe on $\operatorname{Pd}(111)$ and $\operatorname{Pt}(111)$, it is much smaller. For the case of $\mathrm{Xe} / \mathrm{Cu}(111)$, the $\mathrm{Xe}-\mathrm{Cu}$ distance is similar to the sum of radii. Because of the significantly shorter Xe-metal distances for adsorption on $\operatorname{Pd}(111)$ and $\operatorname{Pt}(111)$, it is suggestive of a chemical or covalentlike interaction for these systems.

The same trends and observations however can be explained by polarization and Pauli repulsion effects, as discussed earlier, which we believe are the main interactions at play. Our results however do not exclude that there is some covalent or chemical-bonding-like contribution to the binding.

\section{WHY DOES Xe PREFER LOW-COORDINATED SITES?}

We explain the on-top site preference for Xe adsorption on metal surfaces based on the following observations: (i) The polarization is larger for Xe adatoms in the on-top site, i.e., the induced dipole moment is greater, which contributes a greater attractive interaction between Xe and the metal surfaces. (ii) The Pauli repulsion is weaker for Xe adatoms at the on-top site, giving rise to a smaller contribution to the repulsive potential between $\mathrm{Xe}$ and the metal surfaces, i.e., enhancing the on-top site preference.

The reason for these observations can be largely explained on the basis of the reactivity of the clean surface, at least for the $\mathrm{Cu}(111), \operatorname{Pt}(111)$, and $\mathrm{Pd}(111)$ surfaces. As described earlier, on analyzing the reactivity function of the clean surface, we found that the electronic character at the on-top region is donorlike and in the hollow site regions it is acceptorlike. Thus, for a negative charge, at not too close distance to the surface (or for a dipole moment with negative end pointing toward the metal surface), the on-top site region can easily screen this perturbation by transferring electron density from the on-top region to the hollow site region. Therefore, at intermediate distances the Xe atom becomes polarized more strongly at the on-top geometry, because the 
screening charge at the metal surface can build up more efficiently. This is why the induced dipole moment is greater for Xe in the on-top site. Similarily, at close distances where wave functions start to overlap and Pauli repulsion sets in, the substrate can reduce this repulsion more efficiently at the on-top site by transferring electrons from the on-top site region to the hollow site region, hence, Xe can get closer to the surface. However, for distances very close to the surface, e.g., $1.0 \AA$ below the equilibrium position, this mechanism is not enough to decrease the repulsion and the hollow site becomes more favorable than the on-top site.

\section{CONCLUSION}

In summary, we studied the interaction of Xe atoms with metal surfaces, namely, $\operatorname{Mg}(0001), \mathrm{Al}(111), \mathrm{Ti}(0001)$, $\mathrm{Cu}(111), \mathrm{Pd}(111)$, and $\mathrm{Pt}(111)$, employing DFT with the LDA and PBE functionals and using the all-electron FPLAPW method.

We find that Xe adatoms preferentially bind at low coordination sites, i.e., on-top sites, for all studied metal systems, which is in agreement with available LEED intensity analysis. This finding suggests that on-top site adsorption may be the rule rather than the exception, and it may also be the case for other rare-gas atom and/or metal systems, thus presenting a picture of the interaction between rare-gas atoms and metal surfaces. Indeed our preliminary results indicate that this is true, at least for $\mathrm{Ar}$ and $\mathrm{Kr}$ atoms on $\mathrm{Pd}(111)$ in the $\sqrt{3}$ structure. ${ }^{50}$ The lateral interactions between Xe adatoms do not play a critical role in the adsorption site preference, nor do spin-orbit coupling effects.

The main interactions between Xe and the metal surfaces that determine the on-top site preference were identified as being a site-dependent Pauli repulsion and polarization of the Xe adatoms and topmost substrate atoms. The Pauli repulsion is weaker at the on-top site due to the "donorlike" behavior of the $d_{z^{2}}$-states which allow the Xe adatoms to get closer to the metal surface. A polarization of the Xe adatoms by the metal surface gives rise to an induced dipole moment pointing out of the surface, which results in a decrease of the substrate work function in accord with experimental results.

There is a clear depopulation of the Xe $5 p$-states, mainly, $5 p_{z}$-state, and a population of the lowest unoccupied Xe states, which broaden upon Xe adsorption on the metal surface with tails that extend below the Fermi level becoming partially occupied. This finding is attributed to the fact that the total wave function must be antisymmetric.

The LDA and the PBE functionals predict the same qualitative picture for the interaction between Xe atoms and the metal surfaces. The deviation between the DFT and LEED equilibrium vertical distance is smaller than $10 \%$ for all studied systems, i.e., underestimated (LDA) or overestimated (PBE). The LDA predicts adsorption energies that are closer to the experimental results, for the cases where there are experimental results with which to be compared.

\section{ACKNOWLEDGMENTS}

The authors thank Th. Seyller and R.D. Diehl (LEED analysis) for stimulating discussions.
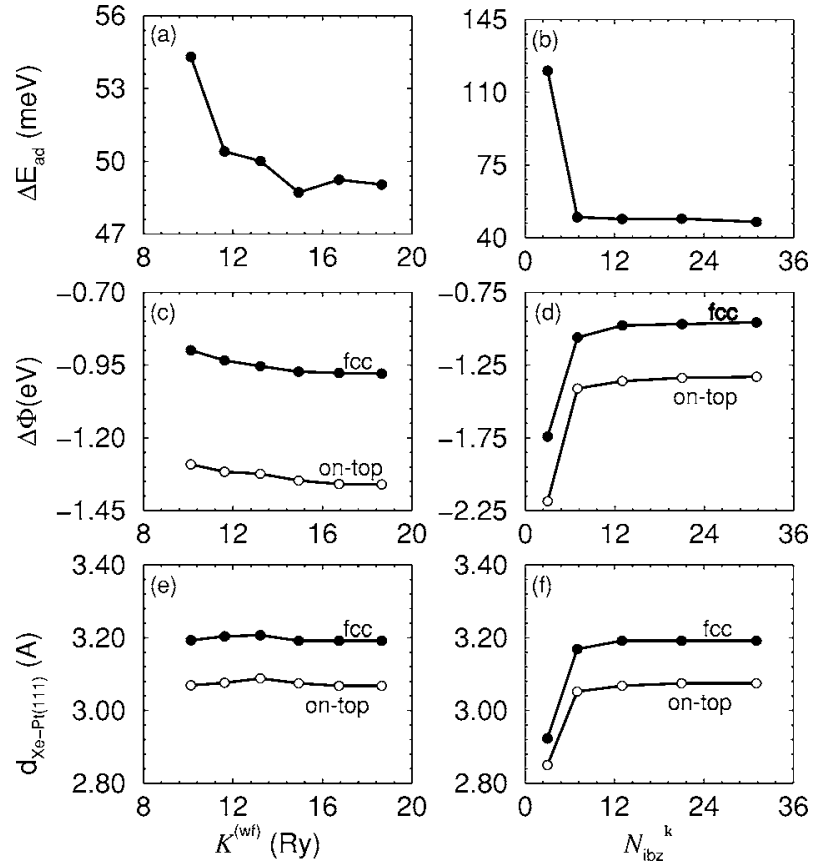

FIG. 11. Convergence tests for Xe adsorption on $\mathrm{Pt}(111)$ in the $\sqrt{3}$ structure with respect to the cutoff energy, $E^{\mathrm{wf}}$, and number of k-points in the IBZ, $N_{\mathrm{ibz}}^{\mathbf{k}}$. (a) and (b) show the relative adsorption energy, $\Delta E_{\mathrm{ad}}^{\mathrm{ft}}$, for Xe in the fcc and on-top sites with respect $E^{\mathrm{wf}}$ and $N_{\mathrm{ibz}}^{\mathbf{k}}$, respectively. (c) and (d) show the induced work function change with respect to the clean surface, $\Delta \Phi$, for Xe in the on-top and fcc sites with respect $E^{\mathrm{wf}}$ and $N_{\mathrm{ibz}}^{\mathrm{k}}$, respectively. (e) and (f) show the equilibrium Xe vertical distance for Xe in the on-top and fcc sites with respect $E^{\mathrm{wf}}$ and $N_{\mathrm{ibz}}^{\mathbf{k}}$, respectively.

\section{APPENDIX}

The present work deals with physisorption systems where the relative energy differences, work function changes, etc., are very small. For example, for $\mathrm{Xe} / \mathrm{Pt}(111)$, the energy difference between the on-top and fcc sites is $49.09 \mathrm{meV}$ (LDA), hence, special care must be taken with respect to the numerical parameters involved in the calculations. Here only the most important convergence tests will be reported. Further results and tests can be found in Ref. 50. Figure 11 shows the convergence of the relative adsorption energy, the induced work function change with respect to the clean surface, and the equilibrium Xe vertical distance for $\mathrm{Xe}$ on $\operatorname{Pt}(111)$ in the $\sqrt{3}$ structure for the on-top and fcc sites as a function of cutoff energy, $E^{\mathrm{wf}}$, and number of $\mathbf{k}$-points in the surface IBZ, $N_{\mathrm{ibz}}^{\mathrm{k}}$.

For cutoff energies from $10.12 \mathrm{Ry}$ to $18.65 \mathrm{Ry}, \Delta E_{\mathrm{ad}}^{\mathrm{ft}}$ changes from $54.31 \mathrm{meV}$ to $49.04 \mathrm{meV}$ (in these calculations $N_{\mathrm{ibz}}^{\mathbf{k}}=13$ ). Most of the calculations for Xe and/or metal were done with $E^{\mathrm{wf}}=14.93 \mathrm{Ry}$ and $16.74 \mathrm{Ry}$, i.e., well converged calculations as seen from Fig. 11(a). For k-points in the IBZ from three k-points to $31 \mathbf{k}$-points, $\Delta E_{\mathrm{ad}}^{\mathrm{ft}}$ changes significantly from $120.38 \mathrm{meV}$ to $47.72 \mathrm{meV} \quad\left(E^{\mathrm{wf}}\right.$ $=16.74 \mathrm{Ry}$ in these calculations). However, it can be seen that for more than seven k-points, the relative adsorption energy is almost constant [see Fig. 11(b)]. Figures $11(\mathrm{c})$ and $11(\mathrm{~d})$ show that $\left(\Delta \Phi^{\mathrm{fcc}}-\Delta \Phi^{\mathrm{on}-\mathrm{top}}\right)$ is almost constant with respect to the cutoff energy and to the number 
of k-points in the IBZ for $N_{\mathrm{ibz}}^{\mathbf{k}} \geqslant 7$.

Figures 11(e) and 11(f) display the equilibrium Xe vertical distance for the on-top and fcc sites as a function of $E^{\mathrm{wf}}$ and $N_{\mathrm{ibz}}^{\mathbf{k}}$ to verify the unexpected behavior of the equilibrium $\mathrm{Xe}$ vertical distance, i.e., that $d_{\mathrm{Xe}-\mathrm{Pt}(111)}^{\text {on-top }}<d_{\mathrm{Xe}-\mathrm{Pt}(111)}^{\mathrm{fcc}}$. Our results show that this result is not an artifact due to unconverged calculations. The present convergence tests show clearly that the on-top site is the most stable site for Xe adsorption on $\mathrm{Pt}(111)$, and the discussed trends do not change with respect to the numerical parameters.
*Present address: Humboldt-Universitaet zu Berlin, Institut fuer Chemie, Arbeitsgruppe Quantenchemie, Unter den Linden 6 D-10099 Berlin, Germany. Electronic address: dasilvaj@chemie.hu-berlin.de

${ }^{1}$ L. W. Bruch, M. W. Cole, and E. Zaremba, Physical Adsorption: Forces and Phenomena (Oxford Science, Oxford, 1997).

${ }^{2}$ M.-C. Desjonquères and D. Spanjaard, Concepts in Surface Science (Springer, New York, 1995).

${ }^{3}$ G. Vidali, G. Ihm, H.-Y. Kim, and M. W. Cole, Surf. Sci. Rep. 12, 133 (1991).

${ }^{4}$ See, e.g., Helium Atom Scattering from Surfaces, edited by E. Hulpke (Springer, Heidelberg, 1992).

${ }^{5}$ K. Wandelt, J. Vac. Sci. Technol. A 2, 802 (1984).

${ }^{6}$ G. K. Horton, Am. J. Phys. 36, 93 (1968).

${ }^{7}$ W. E. Carlos and M. W. Cole, Surf. Sci. 91, 339 (1980).

${ }^{8}$ J. R. Cerdá, P. L. de Andres, F. Flores, and R. Perez, Phys. Rev. B 45, 8721 (1992).

${ }^{9}$ N. W. Ashcroft and N. M. Mermin, Solid State Physics (HoltSaunders International Editions, 1976).

${ }^{10} \mathrm{C}$. Kittel, Introduction to Solid State Physics, 7th ed. (Wiley, New York, 1996).

${ }^{11}$ P. A. Rejto and H. C. Andersen, J. Chem. Phys. 98, 7636 (1993).

${ }^{12}$ G. Hilgers, M. Potthoff, N. Müller, and U. Heinzmann, Surf. Sci. 322, 207 (1995).

${ }^{13}$ M. Potthoff, G. Hilgers, N. Müller, U. Heinzmann, L. Haunert, J. Braun, and G. Borstel, Surf. Sci. 322, 193 (1995).

${ }^{14}$ K. Pussi, J. Smerdon, N. Ferralis, M. Lindroos, R. McGrath, and R. D. Diehl, Surf. Sci. 548, 157 (2004).

${ }^{15}$ J. M. Gottlieb, Phys. Rev. B 42, 5377 (1990).

${ }^{16}$ J. E. Black and A. Janzen, Surf. Sci. 217, 199 (1989).

${ }^{17}$ J. A. Barker, C. T. Retter, and D. S. Bethune, Chem. Phys. Lett. 188, 471 (1992).

${ }^{18}$ Th. Seyller, M. Caragiu, R. D. Diehl, P. Kaukasoina, and M. Lindroos, Chem. Phys. Lett. 291, 567 (1998).

${ }^{19}$ M. Caragiu, Th. Seyller, and R. D. Diehl, Phys. Rev. B 66, 195411 (2002).

${ }^{20}$ B. Narloch and D. Menzel, Chem. Phys. Lett. 290, 163 (1997).

${ }^{21}$ Th. Seyller, M. Caragiu, R. D. Diehl, P. Kaukasoina, and M. Lindroos, Phys. Rev. B 60, 11084 (1999).

${ }^{22}$ M. Caragiu, Th. Seyller, and R. D. Diehl, Surf. Sci. 539, 165 (2003).

${ }^{23}$ Th. Seyller, M. Caragiu, and R. D. Diehl, Surf. Sci. 454, 55 (2000)

${ }^{24}$ P. S. Weiss and D. M. Eigler, Phys. Rev. Lett. 69, 2240 (1992).

${ }^{25}$ D. M. Eigler (private communication).

${ }^{26}$ P. Zeppenfeld, S. Horch, and G. Comsa, Phys. Rev. Lett. 73, 1259 (1994).

${ }^{27}$ K. Kern, R. David, P. Zeppenfeld, and G. Comsa, Surf. Sci. 195, 353 (1988).

${ }^{28}$ F. Brunet, R. Schaub, S. Fédrigo, R. Monot, J. Buttet, and W.
Harbich, Surf. Sci. 512, 201 (2002).

${ }^{29}$ J. E. Müller, Phys. Rev. Lett. 65, 3021 (1990).

${ }^{30}$ N. D. Lang, Phys. Rev. Lett. 46, 842 (1981).

${ }^{31}$ N. D. Lang and J. K. Norskóv, Phys. Rev. B 27, 4612 (1983).

${ }^{32}$ M. Petersen, S. Wilke, P. Ruggerone, B. Kohler, and M. Scheffler, Phys. Rev. Lett. 76, 995 (1996).

${ }^{33}$ A. E. Betancourt and D. M. Bird, J. Phys.: Condens. Matter 12, 7077 (2000).

${ }^{34}$ S. Clarke, G. Bihlmayer, and S. Blügel, Phys. Rev. B 63, 085416 (2001).

${ }^{35}$ J. V. Burda, N. Runeberg, and P. Pyykkö, Chem. Phys. Lett. 288, 635 (1998)

${ }^{36}$ S. Clarke, J. E. Inglesfield, M. Nekovee, and P. K. de Boer, Phys. Rev. Lett. 80, 3571 (1998).

${ }^{37}$ S. Clarke, M. Nekovee, P. K. de Boer, and J. E. Inglesfield, J. Phys.: Condens. Matter 10, 7777 (1998).

${ }^{38}$ P. S. Bagus, V. Staemmler, and C. Wöll, Phys. Rev. Lett. 89, 096104 (2002)

${ }^{39}$ J. L. F. Da Silva, C. Stampfl, and M. Scheffler, Phys. Rev. Lett. 90, 066104 (2003).

${ }^{40}$ P. Hohenberg and W. Kohn, Phys. Rev. 136, B864 (1964).

${ }^{41}$ W. Kohn and L. J. Sham, Phys. Rev. 140, A1133 (1965).

${ }^{42}$ J. P. Perdew and Y. Wang, Phys. Rev. B 45, 13244 (1992).

${ }^{43}$ J. P. Perdew, J. A. Chevary, S. H. Vosko, K. A. Jackson, M. R. Pederson, D. J. Singh, and C. Fiolhais, Phys. Rev. B 46, 6671 (1992).

${ }^{44}$ J. P. Perdew, K. Burke, and M. Ernzerhof, Phys. Rev. Lett. 77, 3865 (1996).

${ }^{45}$ D. J. Singh, Plane Waves, Pseudopotentials and LAPW Method (Kluwer Academic, Boston, Dordrecht, London, 1994).

${ }^{46}$ P. Blaha, K. Schwarz, and J. Luitz, wIEN97, A Full Potential Linearized Augmented Plane Wave Package for Calculating Crystal Properties (Karlheinz Schwarz, Technical University, Wien, Vienna, 1999); updated version of P. Blaha, K. Schwarz, P. Sorantin and S. B. Trickey, Comput. Phys. Commun. 59, 399 (1990).

${ }^{47}$ M. Petersen, F. Wagner, L. Hufnagel, M. Scheffler, P. Blaha, and K. Schwarz, Comput. Phys. Commun. 126, 294 (2000).

${ }^{48}$ R. Dohmen, J. Pichlmeier, M. Petersen, F. Wagner, and M. Scheffler, Comput. Sci. Eng. 3, 18 (2001).

${ }^{49}$ B. Kohler, S. Wilke, M. Scheffler, R. Kouba, and C. AmbroschDraxl, Comput. Phys. Commun. 94, 31 (1996).

${ }^{50}$ J. L. F. Da Silva, Ph.D. thesis (http://edocs.tu-berlin.de/diss/2002/ dasilva_juarez.htm or http://www.fhi-berlin.mpg.de/th/ pub02.html), Technical University Berlin, Berlin, Germany, 2002.

${ }^{51}$ F. Wagner, Th. Laloyaux, and M. Scheffler, Phys. Rev. B 57, 2102 (1998).

${ }^{52}$ H. J. Monkhorst and J. D. Pack, Phys. Rev. B 13, 5188 (1976).

${ }^{53}$ M. G. Gillan, J. Phys.: Condens. Matter 1, 689 (1989).

${ }^{54}$ F. D. Murnaghan, Proc. Natl. Acad. Sci. U.S.A. 50, 697 (1944). 
${ }^{55}$ M. Fuchs, L. L. F. Da Silva, C. Stampfl, J. Neugebauer, and M. Scheffler, Phys. Rev. B 65, 245212 (2002).

${ }^{56} \mathrm{~S}$. Å. Lindgren, L. Walldén, J. Rundgren, and P. Westrin, Phys. Rev. B 29, 576 (1984).

${ }^{57}$ D. L. Adams, H. B. Nielsen, and M. A. Van Hove, Phys. Rev. B 20, 4789 (1979).

${ }^{58}$ H. D. Shih, F. Jona, D. W. Jepsen, and P. M. Marcus, J. Phys. C 9, 1405 (1976).

${ }^{59}$ M. E. Grillo, C. Stampfl, and W. Berndt, Surf. Sci. 317, 84 (1994).

${ }^{60}$ Ismail, Ph. Hofmann, A. P. Baddorf, and E. W. Plummer, Phys. Rev. B 66, 245414 (2002).

${ }^{61}$ C. Stampfl, M. Scheffler, H. Over, J. Burchhardt, M. Nielsen, D. L. Adams, and W. Moritz, Phys. Rev. B 49, 4959 (1994).

${ }^{62}$ P. Zeppenfeld, Physics of Covered Solid Surfaces, Börnstein, New Series, Group III, Vol. 42: Numerical data and functional relationships in science and technology, Subvol. A: Adsorbed layers on surfaces (Springer-Verlag, Berlin, 2001), p. 67.

${ }^{63}$ J. Hölzl, F. K. Schulte, and H. Wagner, "Work function of metals," in Solid State Physics, Springer Tracts Mod. Phys., Vol. 85 (Springer, Berlin, 1979).

${ }^{64}$ K. Wandelt and J. E. Hulse, J. Chem. Phys. 80, 1340 (1984).

${ }^{65}$ S. Horch, P. Zeppenfeld, and G. Comsa, Surf. Sci. 311, 908 (1995).

${ }^{66}$ J. Ellis, A. P. Graham, and J. P. Toennies, Phys. Rev. Lett. 82,
5072 (1999).

${ }^{67}$ E. J. J. Kirchner, A. W. Kleyn, and E. J. Baerends, J. Chem. Phys. 101, 9155 (1994).

${ }^{68}$ L. W. Bruch, Phys. Rev. B 64, 033407 (2001).

${ }^{69}$ M. Scheffler and C. Stampfl, "Theory of adsorption on metal substrates," in Electronic Structure, Vol. 2 of Handbook of Surface Science, edited by K. Horn and M. Scheffler (Elsevier, Amsterdam, 1999), p. 286.

${ }^{70}$ P. R. Antoniewicz, Phys. Rev. Lett. 32, 1424 (1974).

${ }^{71}$ E. Wimmer, A. J. Freeman, J. R. Hiskes, and A. M. Karo, Phys. Rev. B 28, 3074 (1983).

${ }^{72}$ L. D. Schmidt and R. Gomer, J. Chem. Phys. 45, 1605 (1966).

${ }^{73}$ L. W. Swanson and R. W. Strayer, J. Chem. Phys. 48, 2421 (1968).

${ }^{74}$ Z. Sidorski, I. Pelly, and R. Gomer, J. Chem. Phys. 50, 2382 (1969).

${ }^{75}$ K. Wandelt and B. Gumhalter, Surf. Sci. 140, 355 (1984).

${ }^{76}$ D. M. Eigler, P. S. Weiss, E. K. Schweizer, and N. D. Lang, Phys. Rev. Lett. 66, 1189 (1991).

${ }^{77}$ K. Fukui, T. Yonezawa, and H. Shingu, J. Chem. Phys. 20, 722 (1952).

${ }^{78}$ K. Fukui, Science 218, 747 (1982).

${ }^{79}$ S. Wilke, M. H. Cohen, and M. Scheffler, Phys. Rev. Lett. 77, 1560 (1996). 